\title{
Finite time estimation through a continuous-discrete observer
}

\author{
Frédéric Mazenc $^{1}(1)$ | Saeed Ahmed ${ }^{2}$ (i) $\mid$ Michael Malisoff $^{3}$ (i)
}

${ }^{1}$ Inria, the Laboratoire des Signaux et Systèmes (L2S), CNRS UMR 8506, Centre National de la Recherche Scientifique, CentraleSupélec, Université Paris-Sud, Gif-sur-Yvette, France

${ }^{2}$ Department of Electrical and Electronics Engineering, Graduate School of Engineering and Science, Bilkent University, Ankara, Turkey

${ }^{3}$ Department of Mathematics, Louisiana State University, Baton Rouge, Louisiana

\section{Correspondence}

Frédéric Mazenc, Inria, the Laboratoire des Signaux et Systèmes (L2S), CNRS UMR 8506, Centre National de la Recherche Scientifique, CentraleSupélec, Université Paris-Sud, 91192 Gif-sur-Yvette, France. Email:

frederic.mazenc@12s.centralesupelec.fr

\section{Funding information}

PHC Bosphore 2016 France-Turkey, Grant/Award Number: 35634QM; The Scientific and Technological Research Council of Turkey (TÜBITAK), Grant/Award Number: EEEAG-115E820; US National Science Foundation, Grant/Award Number: 1408295 and 1711299

\section{1 | INTRODUCTION}

State estimation of nonlinear systems from output measurements is a basic concern in robotics, ${ }^{1}$ chemical and biochemical processes, ${ }^{2}$ biomedical systems, ${ }^{3}$ communication systems, ${ }^{4}$ automotive systems, ${ }^{5}$ networked control systems, ${ }^{6}$ and many other fields. Due to this strong motivation, various techniques to achieve state observation of nonlinear systems have been discussed in the literature. These techniques have included canonical form observers, ${ }^{7}$ high-gain observers (as in the works of Hammouri et al, ${ }^{8}$ Khalil and Praly, ${ }^{9}$ and Zemouche and Boutayeb ${ }^{10}$ ), Lyapunov-based observers (as in the works of Freidovich and Khaliil ${ }^{11}$ and Kazantzis and Kravaris ${ }^{12}$ ), and extended Kalman and Luenberger observers (as in the works of Wang et $\mathrm{al}^{13}$ and $\mathrm{Zeitz}^{14}$ ).

The aforementioned observer design techniques have the common disadvantage that they only ensure asymptotic convergence of the estimation error to zero, whereas finite time convergence of estimation errors to zero is often desirable for control and supervision purposes; see the works of Du et $\mathrm{al}^{15}$ and Menhold et $\mathrm{al}^{16,17}$; and for more motivation for finite time control, see the work of Song et al. ${ }^{18}$ One can distinguish between two broad families of finite time converging observers, namely, the families composed of nonsmooth observers without delay and the family of the smooth observers with delays.

\begin{abstract}
Summary
We study two broad classes of nonlinear time-varying continuous-time systems with outputs. For the first class, we build an observer in the case where a state dependent disturbance affects the linear approximation. When the disturbances are the zero functions, our observer provides exact values of the state at all times larger than a suitable finite time, and it provides an approximate estimate when there are nonzero disturbances, so our observers are called finite time observers. We use this construction, which is of interest for its own sake, to design a globally exponentially stabilizing dynamic output feedback for a family of nonlinear systems whose outputs are only available on some finite time intervals. Our simulations illustrate the efficacy of our methods.
\end{abstract}

\section{KEYWORDS}

observer, output feedback, stabilization, switched system 
Nonsmooth finite time converging observers have been proposed, for instance, in other works. ${ }^{4,15,19-21}$ The main drawback of nonsmooth finite time observers is that their lack of smoothness may generate poor robustness performance, but they have no delay, which is an advantage because the presence of a delay may complicate the implementation of an observer. Another possible drawback is due to the fact that the time of convergence of each trajectory depends on its initial condition. This is not the case for the observers that use artificial delays, for which their instant of convergence of the solutions is independent of the initial conditions and can be rendered as small as desired by the selection of a parameter (called the artificial delay). Finite time converging smooth observer designs have been introduced more recently. They were first presented in the work of Engel and Kreisselmeier, ${ }^{22}$ which was only applicable to linear time-invariant systems; see also the work of Raff and Allgower ${ }^{23}$ for finite time observers for linear time-invariant systems. An extension of the design presented in the work of Engel and Kreisselmeier ${ }^{22}$ was carried out in the works of Menhold et al ${ }^{16,17}$ for linear time-varying systems and nonlinear systems in observer canonical (normal) form, respectively. A generalized finite time converging observer design technique for nonlinear systems was proposed in the work of Sauvage et $\mathrm{al}^{24}$ and was applicable to noncanonical form nonlinear systems as well. This approach was developed through a Lyapunov-based observer from the work of Kazantzis and Kravaris. ${ }^{12}$

The preceding finite time observer design approaches were carried out without considering disturbances in the measurements or dynamics. Such disturbances are usually present in practical applications and they affect measurements and can be state dependent. Motivated by this fact, a finite time state estimation algorithm for nonlinear systems with bounded and time-varying disturbances in the dynamics and measurements was recently proposed in the work of Mazenc et al. ${ }^{25}$ The design approach used in the work of Mazenc et $\mathrm{al}^{25}$ is similar to that of Sauvage et al. ${ }^{24}$ Other issues pertaining to finite time converging observer design for nonlinear systems have been discussed in the works of Menard et al, ${ }^{20}$ Shen and Huang, ${ }^{26}$ and Shen and Xia. ${ }^{27}$

The present paper has two main objectives. Our first aim is to complement the works of Sauvage et al ${ }^{24}$ and Mazenc et $\mathrm{a}^{25}$ by proposing a finite time converging observer design for Lipschitz nonlinear systems of the form

$$
\left\{\begin{array}{l}
\dot{x}(t)=[A+\epsilon(t, x(t))] x(t)+f(t, y(t), u(t)) \\
y(t)=C x(t)
\end{array}\right.
$$

where $A \in \mathbb{R}^{n \times n}$ and $C \in \mathbb{R}^{q \times n}$ are constant matrices, the state $x$ is valued in $\mathbb{R}^{n}$, the input $u$ is valued in $\mathbb{R}^{p}$, the output $y$ is valued in $\mathbb{R}^{q}, f$ is a nonlinear function that is assumed to be locally Lipschitz with respect to $y$ and piecewise continuous in its other arguments, the locally Lipschitz function $\epsilon:[0,+\infty) \times \mathbb{R}^{n} \rightarrow \mathbb{R}^{n \times n}$ can represent a disturbance, and the dimensions are arbitrary. Systems of the form (1) arise in many engineering contexts, eg, in the modeling of vibrations of elastic membranes; see our examples in the illustrations section.

The key difference between the nonlinear systems in the works of Sauvage et $\mathrm{al}^{24}$ and Mazenc et $\mathrm{al}^{25}$ and (1) is the presence of the function $\epsilon$. This disturbance significantly increases the difficulty of constructing a finite time observer, since it makes it impossible to apply the approaches in the works of Sauvage et $\mathrm{al}^{24}$ and Mazenc et $\mathrm{al}^{25}$ to (1) and it seems that there is no direct way to extend them to (1). The nonlinear term $\epsilon$ is worth considering because (i) disturbances of this type often affect systems and (ii) this term will enable us to use our finite time observer to construct dynamic output feedback for a broad family of nonlinear time-varying systems with temporary loss of output measurements. Very few works design finite time observers for Lipschitz nonlinear systems; see, eg, the work of Shen et $\mathrm{al}^{21}$ for a finite time observer for Lipschitz nonlinear systems under homogeneity conditions that are not required here. An advantage of our observer approach is that the finite convergence time in our method for (1) is independent of the initial state.

Our dynamic output feedback design for our class of nonlinear systems with temporary loss of output measurements is motivated by the fact that, in many engineering applications, the state is not available for measurement, and the output measurements are only available intermittently, meaning there may be intervals during which there is no output measurement, eg, due to communication failures in GPS-denied environments. Our strategy has the following steps. We use an assumption that is inspired by the works of Yan et $\mathrm{al}^{28,29}$ and our finite time observer design to construct a dynamic output feedback through a continuous-discrete observer; see, eg, the work of Mazenc et al ${ }^{30}$ for continuous-discrete observers when the output values are available at all times instead of being intermittent. Our dynamic output feedback globally exponentially stabilizes the origin of the nonlinear systems with a temporary loss of measurements. To the best of our knowledge, the stabilization problem we described with temporary loss of output measurements had remained unsolved in the literature, even in the case of linear systems, so our proposed tools are of considerable independent interest.

Throughout the sequel, the notation will be simplified whenever no confusion can arise from the context. The dimensions of our Euclidean spaces are arbitrary unless otherwise noted. The Euclidean norm in $\mathbb{R}^{a}$ and the induced norm of 
matrices are denoted by $|\cdot|$, and we assume that the initial times for our solutions are 0 . We define $X_{t}$ by $X_{t}(s)=X(t+s)$ for all choices of $X, s \leq 0$, and $t \geq 0$ for which the equality is defined. Let $I$ denote an identity matrix of any dimension. A matrix is called Schur stable provided its spectral radius $\sigma$ satisfies $\sigma<1$. A constant matrix is called positive provided all of its entries are positive. Let $|\cdot|_{\infty}$ denote the sup norm of any matrix valued function over its entire domain.

\section{2 | THREE LEMMAS}

The following lemmas will be used to prove our main results. For their proofs, see Appendices B and D.

Lemma 1. Let $M \in \mathbb{R}^{n \times n}$ be an invertible matrix. Let $N \in \mathbb{R}^{n \times n}$ be a matrix. Let $\bar{n}$ and $\bar{m}$ be two constants such that $\left|M^{-1}\right| \leq \bar{m}$ and $|N| \leq \bar{n}$. Assume that

$$
\bar{m} \bar{n}<1 \text {. }
$$

Then, the matrix $M+N$ is invertible and

$$
\left|(M+N)^{-1}-M^{-1}\right| \leq \frac{\bar{m}^{2} \bar{n}}{1-\bar{m} \bar{n}}
$$

is satisfied.

Lemma 2. Let $\mathcal{A} \in \mathbb{R}^{n \times n}$ be a constant matrix. Consider the system

$$
\dot{\zeta}(t)=[\mathcal{A}+\mathcal{E}(t)] \zeta(t),
$$

where $\zeta$ is valued in $\mathbb{R}^{n}$ and $\mathcal{E}:[0,+\infty) \rightarrow \mathbb{R}^{n \times n}$ is a bounded locally Lipschitz function. Let $\phi$ denote the fundamental solution of system (4). Then, for all $t_{1} \in \mathbb{R}$ and $t_{2} \in \mathbb{R}$ such that $t_{1} \geq t_{2}$, the inequality

$$
\left|\phi\left(t_{1}, t_{2}\right)-e^{\mathcal{A}\left(t_{1}-t_{2}\right)}\right| \leq|\mathcal{E}|_{\infty}\left(t_{1}-t_{2}\right) e^{\left(|\mathcal{A}|+|\mathcal{E}|_{\infty}\right)\left(t_{1}-t_{2}\right)}
$$

is satisfied.

Lemma 3. Let

$$
\mathcal{M}=\left[\begin{array}{ll}
\alpha & \beta \\
\gamma & \omega
\end{array}\right]
$$

be a positive Schur stable matrix. Then, for each constant $\mathcal{N} \geq 0$, we can find constants $c_{i}>0$ for $i=1$ and 2 and $k \in \mathbb{N}$ such that for all piecewise continuous functions $z_{i}:[0,+\infty) \rightarrow[0,+\infty)$ for $i=1,2$ that satisfy

$$
\begin{aligned}
& z_{1}(t) \leq \alpha \sup _{s \in[t-\mathcal{N}, t]} z_{1}(s)+\beta \sup _{s \in[t-\mathcal{N}, t]} z_{2}(s) \\
& z_{2}(t) \leq \gamma \sup _{s \in[t-\mathcal{N}, t]} z_{1}(s)+\omega \sup _{s \in[t-\mathcal{N}, t]} z_{2}(s)
\end{aligned}
$$

for all $t \geq \mathcal{N}$ and that have finite left limits $z_{i}(t-)$ at each point $t \geq 0$ for $i=1$ and 2 , we have $|z(t)| \leq$ $c_{1} e^{-c_{2} t} \sup _{s \in[0, k \mathcal{N}]}|z(s)|$ for all $t \geq k \mathcal{N}$.

\section{3 | FINITE TIME OBSERVER}

\section{1 | Statement of result}

In this section, we complement the works of Sauvage et $\mathrm{a}^{24}$ and Mazenc et $\mathrm{al}^{25}$ where a finite time observer is provided, by allowing the more general class of systems (1) for general choices of the state dependent uncertainties $\epsilon$. We assume the following.

Assumption 1. The function $f$ in (1) is locally Lipschitz with respect to $y$ and $u$ and piecewise continuous with respect to $t$. The pair $(A, C)$ is observable and (1) is forward complete. The function $\epsilon$ is bounded and locally Lipschitz. Finally, $u$ is piecewise continuous and locally bounded. 
When Assumption 1 is satisfied, we can use lemma 1 in the work of Mazenc et al $^{25}$ to show that the observability of $(A, C)$ implies that we can select a matrix $L \in \mathbb{R}^{n \times q}$ with the following property: For each constant $\bar{\tau}>0$, there is a constant $\tau \in(0, \bar{\tau})$ such that with the choice $F=A+L C$, the matrix

$$
E(\tau)=e^{-A \tau}-e^{-F \tau}
$$

is invertible. This follows from an analytic continuity argument by first using lemma 1 in the work of Mazenc et $\mathrm{al}^{25}$ to find a constant $\tau_{a}>0$ and a matrix $L$ such that (7) is invertible with the choices $F=A+L C$ and $\tau=\tau_{a}$, and then noting that, if there were a $\bar{\tau} \in\left(0, \tau_{a}\right)$ such that $\operatorname{det}(E(r))=0$ for all $r \in(0, \bar{\tau})$, then real analyticity of $\operatorname{det}(E(r))$ as a function of $r$ would also give the contradiction $\operatorname{det}\left(E\left(\tau_{a}\right)\right)=0$. Our final assumption is as follows.

Assumption 2. There exist a positive constant $\tau$ and a constant matrix $L$ such that (i) the matrix $E(\tau)$ defined in (7) with the choice $F=A+L C$ is invertible and (ii) the bound

$$
|\epsilon|_{\infty} \tau\left[e^{\left(|A|+|\epsilon|_{\infty}\right) \tau}+e^{\left(|F|+|\epsilon|_{\infty}\right) \tau}\right]\left|E^{-1}(\tau)\right|<1
$$

is satisfied.

We are ready to state and prove the following result.

Theorem 1. Let system (1) satisfy Assumptions 1 and 2. Let

$$
\hat{x}(t)=E^{-1}(\tau) \int_{t-\tau}^{t}\left[\left(e^{(t-\tau-\ell) A}-e^{(t-\tau-\ell) F}\right) f(\ell, y(\ell), u(\ell))+e^{(t-\tau-\ell) F} L y(\ell)\right] \mathrm{d} \ell
$$

for all $t \geq \tau$, where $E, F$, and $\tau$ are from Assumption 2. Then, in terms of the constants

$$
c(\tau)=|\epsilon|_{\infty}\left(e^{\left(|A|+|\epsilon|_{\infty}\right) \tau}+e^{\left(|F|+|\epsilon|_{\infty}\right) \tau}\right)\left[J(\tau)+\left(|\epsilon|_{\infty} J(\tau)+\left|E^{-1}(\tau)\right|\right) \tau\right]
$$

and

$$
J(\tau)=\frac{\tau\left[e^{\left(|A|+|\epsilon|_{\infty}\right) \tau}+e^{\left(|F|+|\epsilon|_{\infty}\right) \tau}\right]\left|E^{-1}(\tau)\right|^{2}}{1-|\epsilon|_{\infty} \tau\left[e^{\left(|A|+|\epsilon|_{\infty}\right) \tau}+e^{\left(|F|+|\epsilon|_{\infty}\right) \tau}\right]\left|E^{-1}(\tau)\right|},
$$

we have

$$
|\hat{x}(t)-x(t)| \leq c(\tau) \int_{t-\tau}^{t}(|f(\ell, y(\ell), u(\ell))|+|L||y(\ell)|) \mathrm{d} \ell
$$

for all $t \geq \tau$ along all maximal solutions of system (1).

Remark 1. In general, $\tau\left[e^{|A| \tau}+e^{|F| \tau}\right]\left|E^{-1}(\tau)\right|$ does not converge to zero when $\tau$ converges to zero, so Assumption 2 is a constraint on $|\epsilon|_{\infty}$. Since inequality (12) holds for all $t \geq \tau$, the function (9) is a finite time observer that gives an approximate value of the solution in finite time and that agrees with the true state variable for all $t \geq \tau$ when $\epsilon=0$. To simplify, and in contrast with the work of Mazenc et al, ${ }^{25}$ we do not assume that there are disturbances in $f$ and $y$. However, an extension to this case can be proved by combining the proof of Theorem 1 with the key ideas of the aforementioned work. ${ }^{25}$

\section{2 | Proof of Theorem 1}

Since system (1) is forward complete, it follows that, for any initial condition, both $x(t)$ and $\hat{x}(t)$ are defined over $[\tau,+\infty)$. Fix any maximal solution $x(t)$ of (1). The proof has two parts. In the first part, we consider the case where $\epsilon$ does not depend on $x$. In the second part, we use the result of the first part to show how the case where $\epsilon$ depends on $x$ can also be handled. To simplify the notation, throughout the proof, we write $f(t, y(t), u(t))$ and $g(t, y(t), u(t))$ as $f(t)$ and $g(t)$, respectively, where $g(t, y(t), u(t))=f(t, y(t), u(t))-L y(t)$ and $L$ is from Assumption 2.

Let $\phi_{1}(t, s)$ and $\phi_{2}(t, s)$ denote the fundamental solutions of the systems

$$
\dot{\xi}_{1}(t)=[A+\epsilon(t)] \xi_{1}(t) \quad \text { and } \quad \dot{\xi}_{2}(t)=[F+\epsilon(t)] \xi_{2}(t),
$$

respectively. Let $\psi_{1}(t, s)=\phi_{1}(t, s)^{-1}$ and $\psi_{2}(t, s)=\phi_{2}(t, s)^{-1}$. It is well known that

$$
\frac{\partial \psi_{1}^{\top}}{\partial t}(t, 0)=-[A+\epsilon(t)]^{\top} \psi_{1}(t, 0)^{\top}
$$


and

$$
\frac{\partial \psi_{2}^{\top}}{\partial t}(t, 0)=-[F+\epsilon(t)]^{\top} \psi_{2}(t, 0)^{\top}
$$

hold for all $t \geq 0$; see appendix C.4 in the work of Sontag. ${ }^{31}$ Let $z_{i}(t)=\psi_{i}(t, 0) x(t)$ for $i=1,2$. Then,

$$
\dot{z}_{1}(t)=\psi_{1}(t, 0)[A+\epsilon(t)] x(t)+\psi_{1}(t, 0) f(t)+\frac{\partial \psi_{1}}{\partial t}(t, 0) x(t)=\psi_{1}(t, 0) f(t)
$$

for all $t \geq 0$. By integrating this equality, we obtain

$$
z_{1}(t)=z_{1}(t-\tau)+\int_{t-\tau}^{t} \psi_{1}(\ell, 0) f(\ell) \mathrm{d} \ell .
$$

Here and in the sequel, all inequalities and equalities are for all $t \geq \tau$ unless otherwise noted. Then, the semigroup property of the flow map $\phi_{1}$ gives $\phi_{1}(t, t-\tau)=\phi_{1}(t, 0) \phi_{1}^{-1}(t-\tau, 0)$ and $\phi_{1}(t, 0) \phi_{1}^{-1}(\ell, 0)=\phi_{1}(t, \ell)$ for all $\ell \in[t-\tau, t]$, and therefore,

$$
\begin{aligned}
x(t) & =\psi_{1}(t, 0)^{-1} \psi_{1}(t-\tau, 0) x(t-\tau)+\int_{t-\tau}^{t} \psi_{1}(t, 0)^{-1} \psi_{1}(\ell, 0) f(\ell) \mathrm{d} \ell \\
& =\phi_{1}(t, t-\tau) x(t-\tau)+\int_{t-\tau}^{t} \phi_{1}(t, \ell) f(\ell) \mathrm{d} \ell .
\end{aligned}
$$

Observing that

$$
\dot{x}(t)=[F+\epsilon(t)] x(t)+g(t, y(t), u(t))
$$

and using variation of parameters give

$$
x(t)=\phi_{2}(t, t-\tau) x(t-\tau)+\int_{t-\tau}^{t} \phi_{2}(t, \ell) g(\ell) \mathrm{d} \ell .
$$

Left multiplying the second line of (17) and (19) by $\psi_{1}(t, t-\tau)$ and $\psi_{2}(t, t-\tau)$, respectively, and computing the difference of the results give

$$
\begin{aligned}
{\left[\psi_{1}(t, t-\tau)-\psi_{2}(t, t-\tau)\right] x(t) } & =\int_{t-\tau}^{t} \psi_{1}(t, t-\tau) \phi_{1}(t, \ell) f(\ell) \mathrm{d} \ell-\int_{t-\tau}^{t} \psi_{2}(t, t-\tau) \phi_{2}(t, \ell) g(\ell) \mathrm{d} \ell \\
& =\int_{t-\tau}^{t} \psi_{1}(\ell, t-\tau) f(\ell) \mathrm{d} \ell-\int_{t-\tau}^{t} \psi_{2}(\ell, t-\tau) g(\ell) \mathrm{d} \ell,
\end{aligned}
$$

where the second equality used the semigroup property of the $\phi_{i}$ 's. Therefore,

$$
[E(\tau)+Z(t)] x(t)=\int_{t-\tau}^{t} \psi_{1}(\ell, t-\tau) f(\ell) \mathrm{d} \ell-\int_{t-\tau}^{t} \psi_{2}(\ell, t-\tau) g(\ell) \mathrm{d} \ell
$$

holds with $Z(t)=\psi_{1}(t, t-\tau)-\psi_{2}(t, t-\tau)-E(\tau)$ and $E$ defined in (7). From (13)-(14), and by Lemma 2 with the choice $\mathcal{E}=\epsilon$, we deduce that

$$
\begin{aligned}
& \left|\psi_{1}(\ell, t-\tau)-e^{-A(\ell-t+\tau)}\right| \leq \bar{\epsilon}(\ell-t+\tau) e^{(|A|+\bar{\epsilon})(\ell-t+\tau)} \\
& \left|\psi_{2}(\ell, t-\tau)-e^{-F(\ell-t+\tau)}\right| \leq \bar{\epsilon}(\ell-t+\tau) e^{(|F|+\bar{e})(\ell-t+\tau)}
\end{aligned}
$$

for all $\ell \in[t-\tau, t]$, where we set $\bar{\epsilon}=|\epsilon|_{\infty}$ for brevity. It follows by setting $\ell=t$ in (22) that

$$
|Z(t)| \leq \bar{\epsilon} \tau\left[e^{(|A|+\bar{\epsilon}) \tau}+e^{(|F|+\bar{\epsilon}) \tau}\right]
$$

for all $t \geq \tau$. From this inequality and Lemma 1 (applied with $M=E(\tau)$ and $N=Z(t)$ ), we deduce that (8) from Assumption 2 ensures that, for all $t \geq \tau$, the matrix $E(\tau)+Z(t)$ is invertible. In addition, Lemma 1 implies that $\left|(E(\tau)+Z(t))^{-1}-E^{-1}(\tau)\right| \leq \bar{\epsilon} J(\tau)$ for all $t \geq \tau$, where $J$ is the constant defined in (11).

Then, omitting the argument $\tau$ of $E$ to keep our notation simple, (21) gives

$$
\begin{aligned}
x(t)= & {[E+Z(t)]^{-1} \int_{t-\tau}^{t}\left[e^{(t-\tau-\ell) A} f(\ell)-e^{(t-\tau-\ell) F} g(\ell)\right] \mathrm{d} \ell } \\
& +[E+Z(t)]^{-1} \int_{t-\tau}^{t}\left[\left(\psi_{1}(\ell, t-\tau)-e^{(t-\tau-\ell) A}\right) f(\ell)+\left(e^{(t-\tau-\ell) F}-\psi_{2}(\ell, t-\tau)\right) g(\ell)\right] \mathrm{d} \ell .
\end{aligned}
$$


We deduce that $\tilde{x}(t)=\hat{x}(t)-x(t)$ satisfies

$$
\begin{aligned}
|\tilde{x}(t)| \leq & \left|E^{-1}-[E+Z(t)]^{-1}\right| \int_{t-\tau}^{t}\left|e^{(t-\tau-\ell) A} f(\ell)-e^{(t-\tau-\ell) F} g(\ell)\right| \mathrm{d} \ell \\
& +\left|[E+Z(t)]^{-1}\right| \int_{t-\tau}^{t}\left[\left|\psi_{1}(\ell, t-\tau)-e^{(t-\tau-\ell) A}\right||f(\ell)|+\left|e^{(t-\tau-\ell) F}-\psi_{2}(\ell, t-\tau)\right||g(\ell)|\right] \mathrm{d} \ell \\
\leq & \bar{\epsilon} J \int_{t-\tau}^{t}\left|e^{(t-\tau-\ell) A} f(\ell)-e^{(t-\tau-\ell) F} g(\ell)\right| \mathrm{d} \ell \\
& +\left(\bar{\epsilon} J+\left|E^{-1}\right|\right) \int_{t-\tau}^{t}\left[\left|\psi_{1}(\ell, t-\tau)-e^{(t-\tau-\ell) A}\right||f(\ell)|+\left|e^{(t-\tau-\ell) F}-\psi_{2}(\ell, t-\tau)\right||g(\ell)|\right] \mathrm{d} \ell,
\end{aligned}
$$

where we omitted the dependency of $J$ on $\tau$ for brevity. Using (22), and setting $\bar{\epsilon}^{\sharp}=\bar{\epsilon}\left(\bar{\epsilon} J+\left|E^{-1}\right|\right)$, we obtain

$$
\begin{aligned}
|\tilde{x}(t)| \leq & \bar{\epsilon} J \int_{t-\tau}^{t}\left|e^{(t-\tau-\ell) A} f(\ell)-e^{(t-\tau-\ell) F} g(\ell)\right| \mathrm{d} \ell \\
& +\bar{\epsilon}^{\sharp} \int_{t-\tau}^{t}\left[(\ell-t+\tau) e^{(|A|+\bar{\epsilon})(\ell-t+\tau)}|f(\ell)|+(\ell-t+\tau) e^{(|F|+\bar{\epsilon})(\ell-t+\tau)}|g(\ell)|\right] \mathrm{d} \ell \\
\leq & \bar{\epsilon} J \int_{t-\tau}^{t}\left[e^{\tau|A|}|f(\ell)|+e^{\tau|F|}|f(\ell)-L y(\ell)|\right] \mathrm{d} \ell \\
& +\bar{\epsilon}^{\sharp} \tau \int_{t-\tau}^{t}\left[e^{(|A|+\bar{\epsilon}) \tau}|f(\ell)|+e^{(|F|+\bar{\epsilon}) \tau}|f(\ell)-L y(\ell)|\right] \mathrm{d} \ell \\
\leq & \bar{\epsilon} J \int_{t-\tau}^{t}\left[\left(e^{\tau|A|}+e^{\tau|F|}\right)|f(\ell)|+e^{\tau|F|}|L||y(\ell)|\right] \mathrm{d} \ell \\
& +\bar{\epsilon}^{\sharp} \tau \int_{t-\tau}^{t}\left[\left(e^{(|A|+\bar{\epsilon}) \tau}+e^{(|F|+\bar{\epsilon}) \tau}\right)|f(\ell)|+e^{(|F|+\bar{\epsilon}) \tau}|L||y(\ell)|\right] \mathrm{d} \ell .
\end{aligned}
$$

This concludes the first part of the proof, by our choice (10) of the constant $c(\tau)$.

We now use the preceding result to cover the case where $\epsilon$ depends on both $t$ and $x$ to complete the proof of the theorem. Fix any specific solution of (1), which we denote by $x_{\natural}(t)$. Then, we consider the system

$$
\left\{\begin{array}{l}
\dot{X}(t)=\left[A+\epsilon\left(t, x_{\natural}(t)\right)\right] X(t)+f(t, Y(t), u(t)) \\
Y(t)=C X(t) .
\end{array}\right.
$$

For system (26), $\epsilon\left(t, x_{\natural}(t)\right)$ depends only on $t$ and not on $X$. Moreover, it is bounded by $\bar{\epsilon}$. Thus, from our previous proof, we deduce that, for all solutions $X$ of $(26)$ and for all $t \geq \tau$, we have

$$
|\hat{X}(t)-X(t)| \leq c(\tau) \int_{t-\tau}^{t}(|f(\ell, Y(\ell), u(\ell))|+|L||Y(\ell)|) \mathrm{d} \ell
$$

with $c(\tau)$ as defined in the first part of the proof and with

$$
\hat{X}(t)=E^{-1}(\tau) \int_{t-\tau}^{t}\left[\left(e^{(t-\tau-\ell) A}-e^{(t-\tau-\ell) F}\right) f(\ell, Y(\ell), u(\ell))+e^{(t-\tau-\ell) F} L Y(\ell)\right] \mathrm{d} \ell .
$$

Since $x_{\natural}(t)$ is a solution of (1), it follows that (26) holds with $X=x_{\natural}$. From (27), it follows that

$$
\left|\hat{x}_{\natural}(t)-x_{\natural}(t)\right| \leq c(\tau) \int_{t-\tau}^{t}\left[\left|f\left(\ell, C x_{\natural}(\ell), u(\ell)\right)\right|+|L|\left|C x_{\natural}(\ell)\right|\right] \mathrm{d} \ell
$$

with

$$
\hat{x}_{\natural}(t)=E^{-1}(\tau) \int_{t-\tau}^{t}\left[\left(e^{(t-\tau-\ell) A}-e^{(t-\tau-\ell) F}\right) f\left(\ell, C x_{\natural}(\ell), u(\ell)\right)+e^{(t-\tau-\ell) F} L C x_{\natural}(\ell)\right] \mathrm{d} \ell .
$$

Since $x_{\natural}$ was an arbitrary solution of (1), this concludes the proof of Theorem 1 . 


\section{4 | STABILIZATION OF SYSTEMS WITH TEMPORARY LOSS OF MEASUREMENTS}

\section{1 | Assumptions and statement of result}

Theorem 1 relies on the assumption that the output is available for all $t \geq 0$ and the fact that the matrix $A$ in (1) does not depend on $t$. In this section, we relax these assumptions. We assume that the output is only available on some specific intervals of time, and we will consider systems whose linear approximation at the origin is time-varying even when no disturbance is acting and $y$ and $u$ are set to zero. Under these assumptions, complemented by a stabilizability assumption of ISS type and a mild restriction on $f$, we construct a globally exponentially stabilizing dynamic output feedback with an observer for the system

$$
\dot{x}(t)=[\mathcal{M}(t)+\eta(t, x(t))] x(t)+\mathcal{B}(t) u(t)
$$

with $x$ valued in $\mathbb{R}^{n}$ for any $n \in \mathbb{N}$ and $u$ valued in $\mathbb{R}^{p}$ for any $p \in \mathbb{N}$. The function $u$ represents a control. We assume that there are constants $P>0$ and $\theta \in(0, P)$ such that the $\mathbb{R}^{p}$ valued output

$$
y(t)=\operatorname{Cx}(t)
$$

is only available when $t$ is in the set

$$
S_{P, \theta}=\bigcup_{i \in \mathbb{N}}[i P, i P+\theta] .
$$

We assume the following.

Assumption 3. The functions $\eta, \mathcal{M}$, and $\mathcal{B}$ are locally Lipschitz and bounded, and $\mathcal{M}$ is not the zero function.

For any locally Lipschitz bounded function $\mathcal{Z}:[0, \infty) \rightarrow \mathbb{R}^{n \times p}$, we can therefore fix a constant $\bar{k}>0$ and choose $s$ matrices $\mathcal{A}_{j} \in \mathbb{R}^{n \times n}$ for $j=1,2, \ldots, s$ such that the function

$$
\mathcal{D}(t, j, x)=\mathcal{M}(t)-\mathcal{Z}(t) \mathcal{C}-\mathcal{A}_{j}+\eta(t, x)
$$

possesses the following property: For each $i \in \mathbb{N}$, there is a $j \in\{1, \ldots, s\}$ (depending on $i$ ) such that, for all $t \in[i P, i P+\theta]$ and all $x \in \mathbb{R}^{n}$, the inequality

$$
|\mathcal{D}(t, j, x)| \leq \bar{k}
$$

is satisfied. In terms of the matrix $\mathcal{C}$ from (31), we also assume that the $\mathcal{A}_{i}$ 's from Assumption 3 satisfy the following.

Assumption 4. For all $i \in\{1, \ldots, s\}$, the pair $\left(\mathcal{A}_{i}, \mathcal{C}\right)$ is observable.

Assumption 4 ensures that there are matrices $\mathcal{L}_{i}$ and values $\tau_{i} \in(0, \theta)$ for $i=1$ to $s$ such that each of the matrices

$$
\mathcal{E}_{i}\left(\tau_{i}\right)=e^{-\mathcal{A}_{i} \tau_{i}}-e^{-\mathcal{F}_{i} \tau_{i}}, \quad \text { where } \mathcal{F}_{i}=\mathcal{A}_{i}+\mathcal{L}_{i} \mathcal{C},
$$

is invertible for $i=1, \ldots, s$. This follows from the analytic continuity argument from Section 3.1.

For later use, we introduce the matrices

$$
\mathcal{H}_{i}=\mathcal{E}_{i}^{-1}\left(\tau_{i}\right)
$$

and upper bounds for the matrices $\mathcal{A}_{i}, \mathcal{F}_{i}, \mathcal{L}_{i}$, and $\mathcal{H}_{i}$ of the form

$$
\left|\mathcal{A}_{i}\right| \leq \overline{\mathcal{A}}, \quad\left|\mathcal{F}_{i}\right| \leq \overline{\mathcal{F}}, \quad\left|\mathcal{L}_{i}\right| \leq \overline{\mathcal{L}}, \quad \text { and } \quad\left|\mathcal{H}_{i}\right| \leq \overline{\mathcal{H}}
$$

for all $i \in\{1, \ldots, s\}$.

Assumption 5. There are a locally Lipschitz function $u_{s}$ and a constant $\mu>0$ such that

$$
\left|u_{s}(t, x)\right| \leq \mu|x|
$$

for all $t \geq 0$ and $x \in \mathbb{R}^{n}$, and constants $\kappa \in(0,1)$ and $\gamma>0$, such that, for any piecewise continuous function $\delta$, all solutions of the system

$$
\dot{x}(t)=[\mathcal{M}(t)+\eta(t, x(t))] x(t)+\mathcal{B}(t) u_{s}(t, x(t)+\delta(t))
$$

satisfy

$$
|x(t)| \leq \kappa|x(s)|+\gamma \sup _{w \in[s, t]}|\delta(w)|
$$

for all $t \geq 0$ and $s \in[t-P-\theta, t-P]$. 
Assumption 5 can be viewed as a generalized Hurwitzness-type condition because, in the specific case where $u_{s}(t, x)=$ $K x$ for some matrix $K$ such that $\mathcal{M}(t)+\eta(t, x(t))+\mathcal{B}(t) K$ is constant and Hurwitz, condition (41) can be checked using variation of parameters. In terms of the constants

$$
\bar{g}=\bar{k}\left(e^{(\overline{\mathcal{A}}+\bar{k}) \bar{\tau}}+e^{(\overline{\mathcal{F}}+\bar{k}) \bar{\tau}}\right)[\mathbb{J}+(\bar{k} \mathbb{J}+\overline{\mathcal{H}}) \bar{\tau}]
$$

and

$$
\mathbb{J}=\frac{\bar{\tau}\left[e^{(\overline{\mathcal{A}}+\bar{k}) \bar{\tau}}+e^{(\overline{\mathcal{F}}+\bar{k}) \bar{\tau}}\right] \overline{\mathcal{H}}^{2}}{1-\bar{k} \bar{\tau}\left[e^{(\overline{\mathcal{A}}+\bar{k}) \bar{\tau}}+e^{(\overline{\mathcal{F}}+\bar{k}) \bar{\tau}}\right] \overline{\mathcal{H}}} \text { and } \bar{\tau}=\max \left\{\tau_{i}: 1 \leq i \leq s\right\},
$$

our final assumption is as follows.

Assumption 6. The inequality

$$
\bar{k} \bar{\tau}\left[e^{(\overline{\mathcal{A}}+\bar{k}) \bar{\tau}}+e^{(\overline{\mathcal{F}}+\bar{k}) \bar{\tau}}\right] \overline{\mathcal{H}}<1
$$

is satisfied. Moreover, with the choices

$$
\bar{p}_{1}=\bar{g}|\mathcal{B}|_{\infty} \mu \quad \text { and } \quad \bar{p}_{2}=\bar{g}\left(|\mathcal{B}|_{\infty} \mu+\left(\overline{\mathcal{L}}+|\mathcal{Z}|_{\infty}\right)|\mathcal{C}|\right),
$$

the matrix

$$
\bar{S}=\left[\begin{array}{cc}
\bar{p}_{1} e^{|\mathcal{M}|_{\infty} P} \bar{\tau} & \bar{p}_{2} e^{|\mathcal{M}|_{\infty} P} \bar{\tau}+|\eta|_{\infty} e^{P \mathcal{M} \infty} \frac{e^{|\mathcal{M}|_{\infty} P}-1}{|\mathcal{M}|_{\infty}} \\
\gamma & \kappa
\end{array}\right]
$$

is Schur stable.

Defining the switching signal $\sigma:[0,+\infty) \rightarrow\{1, \ldots, s\}$ by $\sigma(t)=j$ for all $t \in[i P,(i+1) P)$ and integers $i \geq 0$, where $j \in\{1, \ldots, s\}$ is any integer such that $|\mathcal{D}(t, j, x)| \leq \bar{k}$ for all $t \in[i P, i P+\theta]$ and all $x \in \mathbb{R}^{n}$, it follows from our choices of $\bar{k}$ and the $\mathcal{A}_{i}$ 's that, for all $t \in S_{P, \theta}$ and $x \in \mathbb{R}^{n}$, we have $|\mathcal{D}(t, \sigma(t), x)| \leq \bar{k}$. Notice that system (31) can be rewritten as

$$
\dot{x}(t)=\left[\mathcal{A}_{\sigma(t)}+\mathcal{D}(t, \sigma(t), x(t))\right] x(t)+\mathcal{Z}(t) \mathcal{C} x(t)+\mathcal{B}(t) u(t) .
$$

Thus, the following theorem can be interpreted as a result for switched systems.

Theorem 2. Let system (31) satisfy Assumptions 3 to 6 and consider the continuous-discrete system

$$
\begin{aligned}
& \dot{\hat{x}}(t)=\mathcal{M}(t) \hat{x}(t)+\mathcal{B}(t) u_{S}(t, \hat{x}(t)), \quad t \in\left[t_{i}, t_{i+1}\right) \\
& \hat{x}\left(t_{i}\right)=\mathcal{H}_{\sigma\left(t_{i}\right)}\left(\tau_{i}\right) \int_{t_{i}-\tau_{i}}^{t_{i}}\left[\mathcal{E}\left(t_{i}-\tau_{i}-\ell, \sigma\left(t_{i}\right)\right)\left(\mathcal{Z}(\ell) y(\ell)+\mathcal{B}(\ell) u_{s}(\ell, \hat{x}(\ell))\right)+e^{\left(t_{i}-\tau_{i}-\ell\right) \mathcal{F}_{\sigma\left(t_{i}\right)}} \mathcal{L}_{\sigma\left(t_{i}\right)} y(\ell)\right] \mathrm{d} \ell,
\end{aligned}
$$

where $\mathcal{E}(m, k)=e^{m \mathcal{A}_{k}}-e^{m F_{k}}, \mathcal{H}_{\sigma\left(t_{i}\right)}$ is the matrix defined in (37) and $t_{i}=i P+\theta$ for integers $i \in \mathbb{N}$. Then, system (31) in closed loop with the dynamic output feedback

$$
u_{s}(t, \hat{x}(t))
$$

is such that the dynamics for $(x, \tilde{x})$ are globally exponentially stable to 0 , where $\tilde{x}(t)=x(t)-\hat{x}(t)$.

Remark 2. Since we chose values $\tau_{i} \in(0, \theta)$, (48) only requires $y(t)$ values at times $t \in S_{P, \theta}$. Theorem 2 implies that the dynamic output feedback (49) asymptotically stabilizes the closed-loop system to 0 . Assumption 3 is inspired by the technique of Yan et $\mathrm{al}^{28,29}$ which makes it possible to stabilize time-varying systems with auxiliary switched systems whose subsystems are time-invariant systems that are affected by disturbances. Notice that, if $|\eta|_{\infty}=0$ and if $\mathcal{M}$ and $\mathcal{Z}$ are continuous and periodic with the same period, then one can have an arbitrarily small constant $\bar{k}$ by choosing a sufficiently large number of matrices $\mathcal{A}_{i}$, which can facilitate satisfying Assumption 6.

Remark 3. Assumption 5 is a stabilizability assumption. Constructing a feedback $u_{s}$ such that (41) is satisfied can be challenging. However, see the works of Malisoff and Mazenc ${ }^{32,33}$ for techniques for constructing feedback controls for nonlinear time-varying systems. The main difference between (31) and the system studied in the work of Mazenc et al ${ }^{30}$ is that $\mathcal{M}$ depends on $t$, but the work of Mazenc et $\mathrm{al}^{30}$ does not provide exponentially stabilizing feedbacks and so does not apply to the problems we study here. 
Remark 4. If $|\eta|_{\infty}=0$ and $\bar{k}$ can be chosen arbitrarily small, then by choosing both $\bar{\tau}$ and $\bar{k}$ sufficiently small, the matrix $\bar{S}$ is Schur stable since $\kappa \in(0,1)$. In this case, there exists a $\bar{\eta}>0$ such that the corresponding matrix $\bar{S}$ is Schur stable if $|\eta|_{\infty} \leq \bar{\eta}$.

Remark 5. The size of the constant $\bar{k}$ depends on $\theta$, and our condition (44) is more easily satisfied when $\bar{k}$ is sufficiently small. Thus, since an arbitrarily small constant $\theta$ can be selected, it may be useful to choose a $\theta$ value resulting in a small constant $\bar{k}>0$.

Remark 6. Theorem 2 can be extended to the case where $C$ in the output depends on $t$. For the sake of simplicity, we do not investigate this case. Notice that $\mathcal{Z}(t)$ can always be chosen identically equal to zero. However, to decrease the conservatism of the approach, it is worth introducing the function $\mathcal{Z}$ that can be freely chosen.

\section{2 | Proof of Theorem 2}

Let $i \geq 0$ be an integer. Bearing in mind (47), we deduce that system (31) in closed loop with $u_{s}(t, \hat{x}(t))$ can be rewritten as

$$
\dot{x}(t)=\left[\mathcal{A}_{\sigma\left(t_{i}\right)}+\mathcal{D}\left(t, \sigma\left(t_{i}\right), x(t)\right)\right] x(t)+\mathcal{Z}(t) \mathcal{C} x(t)+\mathcal{B}(t) u_{s}(t, \hat{x}(t))
$$

for all $t \in[i P,(i+1) P)$ and $i \geq 0$, and our linear growth condition (39) on $u_{s}$ ensures that the closed-loop system is forward complete. Assumptions 3 to 6 imply that, for each choice of $i$, Assumptions 1 and 2 are satisfied by

$$
\left\{\begin{array}{l}
\dot{x}(t)=\left[\mathcal{A}_{\sigma\left(t_{i}\right)}+\mathcal{D}^{b}\left(t, \sigma\left(t_{i}\right), x(t)\right)\right] x(t)+\mathcal{Z}(t) y(t)+\mathcal{B}(t) u(t) \\
y(t)=\mathcal{C} x(t),
\end{array}\right.
$$

where $u(t)=u_{s}(t, \hat{x}(t))$, and where $\mathcal{D}^{b}$ is any locally Lipschitz function that is equal to $\mathcal{D}$ on $[i P, i P+\theta] \times\left\{\sigma\left(t_{i}\right)\right\} \times \mathbb{R}^{n}$ and that is bounded by $\bar{k}$. (We introduce the function $\mathcal{D}^{b}$ because $\bar{k}$ is not necessarily a global bound on $\mathcal{D}$ but provides the required bound for $t \in[i P, i P+\theta]$.) Therefore, since the $\hat{x}_{i}$ formula from (48) agrees with the $\hat{x}(t)$ formula from (9) for suitable choices of $f$ and other functions from (9), we deduce from Theorem 1 that

$$
\left|\tilde{x}\left(t_{i}\right)\right| \leq \bar{g} \int_{t_{i}-\tau_{i}}^{t_{i}}\left[\left|\mathcal{B}(\ell) u_{s}(\ell, \hat{x}(\ell))\right|+\left(|\mathcal{Z}|_{\infty}+\left|\mathcal{L}_{\sigma\left(t_{i}\right)}\right|\right)|y(\ell)|\right] \mathrm{d} \ell
$$

with $\bar{g}$ defined in (42) and $\tilde{x}=\hat{x}-x$. As an immediate consequence of the bounds (38), we obtain

$$
\left.\left|\tilde{x}\left(t_{i}\right)\right| \leq \bar{g} \int_{t_{i}-\tau_{i}}^{t_{i}}\left[|\mathcal{B}|_{\infty} \mu|\hat{x}(\ell)|+\left(\overline{\mathcal{L}}+|\mathcal{Z}|_{\infty}\right)|\mathcal{C}||x(\ell)|\right] \mathrm{d} \ell \leq \int_{t_{i}-\tau_{i}}^{t_{i}}\left[\bar{p}_{1} \mid \tilde{x}(\ell)\right)\left|+\bar{p}_{2}\right| x(\ell) \mid\right] \mathrm{d} \ell
$$

with $\bar{p}_{1}$ and $\bar{p}_{2}$ defined in (45).

On the other hand, (31) and (48) imply that, for all $t \in\left[t_{i}, t_{i+1}\right)$ and integers $i \geq 0$, we have

$$
\dot{\tilde{x}}(t)=\mathcal{M}(t) \tilde{x}(t)-\eta(t, x(t)) x(t)
$$

when we use the control (49). This gives $|\dot{\tilde{x}}(t)| \leq|\mathcal{M}|_{\infty}|\tilde{x}(t)|+|\eta|_{\infty}|x(t)|$, which we can integrate to obtain

$$
|\tilde{x}(t)| \leq\left(\left|\tilde{x}\left(t_{i}\right)\right|+\int_{t_{i}}^{t}|\eta|_{\infty}|x(\ell)| \mathrm{d} \ell\right)+\int_{t_{i}}^{t}|\mathcal{M}|_{\infty}|\tilde{x}(\ell)| \mathrm{d} \ell .
$$

Hence, Gronwall's inequality gives

$$
\begin{aligned}
|\tilde{x}(t)| & \leq\left(\left|\tilde{x}\left(t_{i}\right)\right|+\int_{t_{i}}^{t}|\eta|_{\infty}|x(\ell)| \mathrm{d} \ell\right) e^{|\mathcal{M}|_{\infty}\left(t-t_{i}\right)} \\
& \leq e^{|\mathcal{M}|_{\infty}\left(t-t_{i}\right)}\left|\tilde{x}\left(t_{i}\right)\right|+|\eta|_{\infty} \int_{t_{i}}^{t}|x(\ell)| e^{|\mathcal{M}|_{\infty}(t-\ell)} e^{|\mathcal{M}|_{\infty}\left(\ell-t_{i}\right)} \mathrm{d} \ell \\
& \leq e^{|\mathcal{M}|_{\infty}\left(t-t_{i}\right)}\left|\tilde{x}\left(t_{i}\right)\right|+|\eta|_{\infty} e^{P|\mathcal{M}|_{\infty}} \int_{t_{i}}^{t}|x(\ell)| e^{|\mathcal{M}|_{\infty}(t-\ell)} \mathrm{d} \ell .
\end{aligned}
$$


We can combine the last inequality in (56) with (53) to obtain

$$
\left.|\tilde{x}(t)| \leq e^{|\mathcal{M}|_{\infty}\left(t-t_{i}\right)} \int_{t_{i}-\bar{\tau}}^{t_{i}}\left[\bar{p}_{1} \mid \tilde{x}(\ell)\right)\left|+\bar{p}_{2}\right| x(\ell) \mid\right] \mathrm{d} \ell+|\eta|_{\infty} e^{P|\mathcal{M}|_{\infty}} \int_{t_{i}}^{t} e^{|\mathcal{M}|_{\infty}(t-\ell)}|x(\ell)| \mathrm{d} \ell
$$

for all $t \in\left[t_{i}, t_{i+1}\right)$, and so,

$$
|\tilde{x}(t)| \leq \bar{p}_{1} e^{|\mathcal{M}|_{\infty}\left(t-t_{i}\right)} \bar{\tau} \sup _{w \in\left[t_{i}-\bar{\tau}, t_{i}\right]}|\tilde{x}(w)|+\left[\bar{p}_{2} e^{|\mathcal{M}|_{\infty}\left(t-t_{i}\right)} \bar{\tau}+|\eta|_{\infty} e^{P|\mathcal{M}|_{\infty}} \frac{e^{|\mathcal{M}|_{\infty} P}-1}{|\mathcal{M}|_{\infty}}\right] \sup _{w \in\left[t_{i}-\bar{\tau}, t\right]}|x(w)|,
$$

where we used the fact that

$$
\int_{t_{i}}^{t} e^{|\mathcal{M}|_{\infty}(t-\ell)}|x(\ell)| \mathrm{d} \ell \leq \frac{1}{|\mathcal{M}|_{\infty}}\left(e^{|\mathcal{M}|_{\infty}\left(t-t_{i}\right)}-1\right) \sup _{w \in\left[t_{i}, t\right]}|x(w)| \leq \frac{1}{|\mathcal{M}|_{\infty}}\left(e^{|\mathcal{M}|_{\infty} P}-1\right) \sup _{w \in\left[t_{i}-\bar{\tau}, t\right]}|x(w)|
$$

for all $t \in\left[t_{i}, t_{i+1}\right)$.

We deduce that, for all $t \geq P+\bar{\tau}$,

$$
|\tilde{x}(t)| \leq \bar{p}_{1} e^{|\mathcal{M}|_{\infty} P} \bar{\tau} \sup _{w \in[t-P-\bar{\tau}, t]}|\tilde{x}(w)|+\left[\bar{p}_{2} e^{|\mathcal{M}|_{\infty} P} \bar{\tau}+|\eta|_{\infty} e^{P|\mathcal{M}|_{\infty}} \frac{e^{|\mathcal{M}|_{\infty} P}-1}{|\mathcal{M}|_{\infty}}\right] \sup _{w \in[t-P-\bar{\tau}, t]}|x(w)| .
$$

System (31) in closed loop with (49) admits the representation

$$
\dot{x}(t)=[\mathcal{M}(t)+\eta(t, x(t))] x(t)+\mathcal{B}(t) u_{s}(x(t)+\tilde{x}(t)) .
$$

From Assumption 5 and the fact that $\bar{\tau} \leq \theta$, it follows that

$$
|x(t)| \leq \kappa|x(t-P-\bar{\tau})|+\gamma \sup _{w \in[t-P-\bar{\tau}, t]}|\tilde{x}(w)|
$$

for all $t \geq P+\bar{\tau}$. By grouping (57) and (59), we obtain

$$
\begin{aligned}
& |\tilde{x}(t)| \leq \bar{p}_{1} e^{|\mathcal{M}|_{\infty} P} \bar{\tau} \sup _{w \in[t-P-\bar{t}, t]}|\tilde{x}(w)|+\left[\bar{p}_{2} e^{|\mathcal{M}|_{\infty} P} \bar{\tau}+|\eta|_{\infty} e^{P|\mathcal{M}|_{\infty}} \frac{e^{|\mathcal{M}|_{\infty} P}-1}{|\mathcal{M}|_{\infty}}\right] \sup _{w \in[t-P-\bar{\tau}, t]}|x(w)| \\
& |x(t)| \leq \kappa \sup _{w \in[t-P-\bar{\tau}, t]}|x(w)|+\gamma \sup _{w \in[t-P-\bar{\tau}, t]}|\tilde{x}(w)|
\end{aligned}
$$

for all $t \geq P+\bar{\tau}$. System (60) is of the form (6) from Lemma 3 (with $\left(z_{1}, z_{2}\right)=(|x(t)|,|\tilde{x}(t)|), \mathcal{N}=P+\bar{\tau}$, and $\mathcal{M}=\overline{\mathcal{S}}$ ), save for the fact that the matrix $\bar{S}$ from (46) is Schur stable but is not necessarily a positive Schur stable matrix (since some of the entries of $\bar{S}$ can be zero). However, we can majorize all of the entries of $\bar{S}$ by positive values, in such a way that the new positive matrix that we obtain is a positive Schur stable matrix. This follows from the continuity of the eigenvalues of a matrix as functions of the entries of the matrix, by increasing the entries of $\bar{S}$ by adding small enough positive constants to the entries. Then, it follows from Lemma 3 that $(x(t), \tilde{x}(t))$ converges exponentially to the origin, which proves the theorem.

\section{5 | ILLUSTRATIONS}

Our classes of dynamics (1) and (31) cover a broad class of systems that were beyond the scope of existing finite time observer approaches. In this section, we illustrate our theorems using the controlled Mathieu equation

$$
\ddot{q}(t)+\left(R_{1}+R_{2} \cos (t)\right) q(t)+u(t)=0
$$

for positive constants $R_{1}$ and $R_{2}$, which arises in the study of vibrations of an elliptic membrane. The Mathieu equation has also been studied in the work of Pearson ${ }^{34}$ to illustrate parameter identification for a certain family of nonlinear and time-varying systems using data over a limited time interval. See also the work of Insperger and Stépán ${ }^{35}$ for the study of

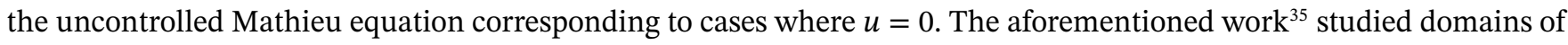
stability and instability including Hopf bifurcations along the boundaries of the domains of stability. 


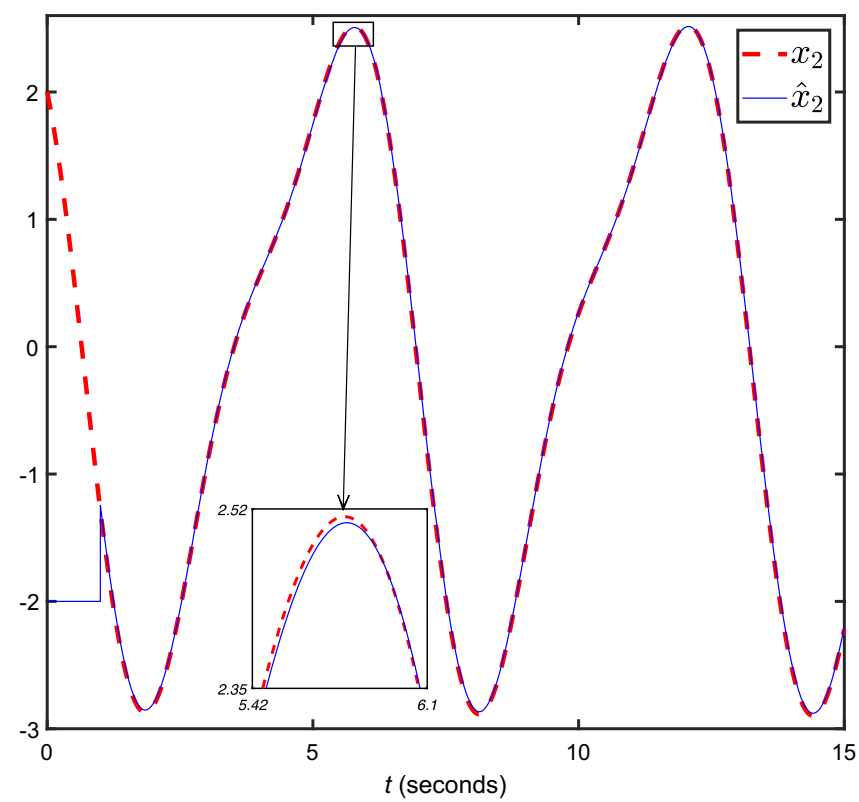

FIGURE 1 Simulations of finite-time observer (9) for (62): component $x_{2}$ and its estimate $\hat{x}_{2}$ [Colour figure can be viewed at wileyonlinelibrary.com]

\section{1 | Illustration of Theorem 1}

The controlled Mathieu Equation (61) can be written as

$$
\left\{\begin{array}{l}
\dot{x}(t)=[A+\epsilon(t, x(t))] x(t)+f(t, y(t), u(t)) \\
y(t)=C x(t)
\end{array}\right.
$$

with

$$
x=\left[\begin{array}{c}
q \\
\dot{q}
\end{array}\right], \quad A=\left[\begin{array}{cc}
0 & 1 \\
-R_{1} & 0
\end{array}\right], \quad C=e_{1}^{\top}, \quad \epsilon(t)=\left[\begin{array}{cc}
0 & 0 \\
-R_{2} \cos (t) & 0
\end{array}\right], \text { and } f(t, y, u)=-e_{2} u,
$$

where $e_{i}$ is the $i$ th standard basis vector for $i=1$ and 2 . We consider the case where only an upper bound on $R_{2}$ is known.

Choosing

$$
L=\left[\begin{array}{c}
0 \\
2 R_{1}
\end{array}\right]
$$

we can satisfy Assumptions 1 and 2 for many choices of the parameters $R_{1}, R_{2}$, and $\tau$. For instance, if we choose $R_{1}=1$, any $R_{2} \in(0,0.024]$, and $\tau=1$, then the matrix $E(\tau)=e^{-A \tau}-e^{-F \tau}$ with the choice $F=A+L C$ has determinant $\operatorname{det}(E(\tau))=0.33254$ and so is invertible, and

$$
|\epsilon|_{\infty} \tau\left[e^{\left(|A|+|\epsilon|_{\infty}\right) \tau}+e^{\left(|F|+|\epsilon|_{\infty}\right) \tau}\right]\left|E^{-1}(\tau)\right|=0.998418<1,
$$

which ensures that Assumptions 1 and 2 are satisfied for any piecewise continuous locally bounded choice of $u$. Then, the finite time observer is provided by (9) from Theorem 1.

To illustrate our result, Figure 1 shows a MATLAB simulation of our finite time observer (9), using an integration algorithm of the model (62) with a semi-implicit integration step of 0.001 and with the initial conditions $x_{1}(0)=x_{2}(0)=2$ and $\hat{x}_{1}(0)=\hat{x}_{2}(0)=-2$, where $u(t)=\sin (2 t)$, and using the preceding choices of $A, f$, and $L$. Since our simulation shows good tracking performance of the estimator $\hat{x}_{2}$ for the state component $x_{2}$ of (62), it helps to illustrate our general theory in the special case of system (62).

\section{2 | Illustration of Theorem 2}

We rewrite the controlled Mathieu equation (61) as

$$
\left\{\begin{array}{l}
\dot{x}(t)=[\mathcal{M}(t)+\eta(t, x(t))] x(t)+\mathcal{B}(t) u(t) \\
y(t)=\mathcal{C} x(t)
\end{array}\right.
$$


with

$$
x=\left[\begin{array}{c}
q \\
\dot{q}
\end{array}\right], \quad \mathcal{M}(t)=\left[\begin{array}{cc}
0 & 1 \\
-R_{1}-R_{2} \cos (t) & 0
\end{array}\right], \quad \mathcal{C}=e_{1}^{\top}, \quad \eta(t, x)=0, \quad \text { and } \mathcal{B}(t)=-e_{2} .
$$

We choose $P=2 \pi$. We consider the case where $R_{1}=1$ and $R_{2}=0.024$, and we apply Theorem 2 with $s=1$, and we use $\tau$ to denote the constant $\tau_{1}$ from Theorem 2. The preceding choices of $\eta, \mathcal{M}$, and $\mathcal{B}$ ensure that Assumption 3 is satisfied. Let

$$
\mathcal{A}_{1}=\left[\begin{array}{cc}
0 & 1 \\
-R_{1}-R_{2} & 0
\end{array}\right] \text { and } \mathcal{Z}(t)=\left[\begin{array}{c}
0 \\
R_{2}(1-\cos (t))
\end{array}\right] .
$$

Then, Assumption 4 is satisfied, and our choice of $\mathcal{Z}$ gives $\mathcal{D}(t, j, x)=\mathcal{M}(t)-\mathcal{Z}(t) \mathcal{C}-\mathcal{A}_{j}+\eta(t, x)=0$. Consequently, our requirements on the functions $D$ are satisfied with $\bar{k}=0$.

We choose

$$
\mathcal{L}_{1}=\left[\begin{array}{c}
0 \\
2 R_{1}
\end{array}\right] \text { and } \mathcal{F}_{1}=\mathcal{A}_{1}+\mathcal{L}_{1} \mathcal{C}=\left[\begin{array}{cc}
0 & 1 \\
R_{1}-R_{2} & 0
\end{array}\right]
$$

Then, the matrix $\mathcal{E}_{1}(\tau)=e^{-\mathcal{A}_{1} \tau}-e^{-\mathcal{F}_{1} \tau}$ is

$$
\mathcal{E}_{1}(\tau)=\left[\begin{array}{cc}
\cos \left(\omega_{1} \tau\right) & -\frac{1}{\omega_{1}} \sin \left(\omega_{1} \tau\right) \\
\omega_{1} \sin \left(\omega_{1} \tau\right) & \cos \left(\omega_{1} \tau\right)
\end{array}\right]-\left[\begin{array}{cc}
\cosh \left(\omega_{2} \tau\right) & -\frac{1}{\omega_{2}} \sinh \left(\omega_{2} \tau\right) \\
-\omega_{2} \sinh \left(\omega_{2} \tau\right) & \cosh \left(\omega_{2} \tau\right)
\end{array}\right],
$$

where $\omega_{1}=\sqrt{R_{1}+R_{2}}$ and $\omega_{2}=\sqrt{R_{1}-R_{2}}$. This can be checked by noting that the terms in the difference in (70) are the fundamental matrix solutions of $\dot{M}=-\mathcal{A}_{1} M$ and $\dot{M}=-\mathcal{F}_{1} M$, respectively. Thus,

$$
\mathcal{E}_{1}(\tau)=\left[\begin{array}{cc}
\cos \left(\omega_{1} \tau\right)-\cosh \left(\omega_{2} \tau\right) & -\frac{1}{\omega_{1}} \sin \left(\omega_{1} \tau\right)+\frac{1}{\omega_{2}} \sinh \left(\omega_{2} \tau\right) \\
\omega_{1} \sin \left(\omega_{1} \tau\right)+\omega_{2} \sinh \left(\omega_{2} \tau\right) & \cos \left(\omega_{1} \tau\right)-\cosh \left(\omega_{2} \tau\right)
\end{array}\right] .
$$

Then,

One can easily check that $\operatorname{det} \mathcal{E}_{1}(0)=0$ and

$$
\operatorname{det} \mathcal{E}_{1}(\tau)=2\left[1-\cos \left(\omega_{1} \tau\right) \cosh \left(\omega_{2} \tau\right)-\frac{R_{2}}{\omega_{1} \omega_{2}} \sin \left(\omega_{1} \tau\right) \sinh \left(\omega_{2} \tau\right)\right] .
$$

$$
\begin{aligned}
\frac{\mathrm{d}}{\mathrm{d} \tau} \operatorname{det} \mathcal{E}_{1}(\tau)=2 R_{1}( & \frac{1}{\sqrt{R_{1}+R_{2}}} \sin \left(\sqrt{R_{1}+R_{2}} \tau\right) \cosh \left(\sqrt{R_{1}-R_{2}} \tau\right) \\
& \left.-\frac{1}{\sqrt{R_{1}-R_{2}}} \cos \left(\sqrt{R_{1}+R_{2}} \tau\right) \sinh \left(\sqrt{R_{1}-R_{2}} \tau\right)\right)>0
\end{aligned}
$$

for all

$$
\tau \in\left(0, \frac{\pi}{2 \sqrt{1.024}}\right)
$$

This follows by noting that (72) is equivalent to positivity of

$$
S(\tau)=\frac{1}{\omega_{1}} \tan \left(\omega_{1} \tau\right)-\frac{1}{\omega_{2}} \tanh \left(\omega_{2} \tau\right)
$$

when (73) holds, which follows because $S(0)=0$ and $S^{\prime}>0$ on this interval. It follows that $\mathcal{E}_{1}(\tau)$ is invertible when $\tau \in(0, \pi / 4]$. To check Assumption 5, we select the feedback $u_{S}(t, x)=\left[1-R_{1}-R_{2} \cos (t)\right] x_{1}+2 x_{2}$. Then, the system that corresponds to (40) is $\dot{x}(t)=G x(t)-e_{2} \delta_{a}(t)$, with

$$
G=\left[\begin{array}{cc}
0 & 1 \\
-1 & -2
\end{array}\right] \text { and } \delta_{a}(t)=\left(1-R_{1}-R_{2} \cos (t)\right) \delta_{1}(t)+2 \delta_{2}(t) .
$$

One can check that, for all $\ell \geq 0$, we have

$$
e^{G \ell}=e^{-\ell}\left[\begin{array}{cc}
1+\ell & \ell \\
-\ell & 1-\ell
\end{array}\right]
$$

and therefore,

$$
\left|e^{G \ell}\right|=e^{-\ell}\left|I+\left[\begin{array}{cc}
\ell & \ell \\
-\ell & -\ell
\end{array}\right]\right| \leq e^{-\ell}(1+2 \ell)
$$



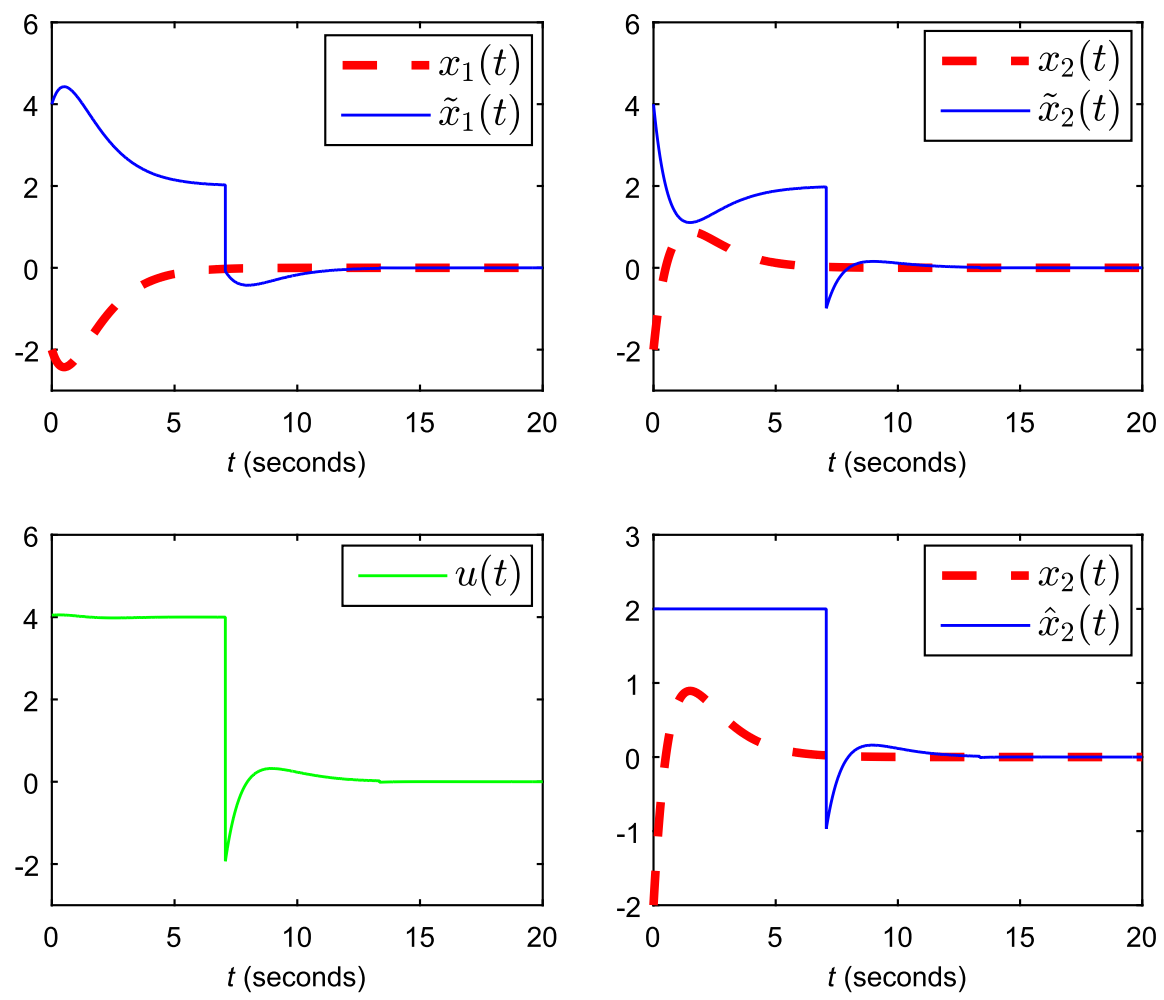

FIGURE 2 Simulation using controller (49) and continuous-discrete observer (48) for (66) [Colour figure can be viewed at wileyonlinelibrary.com]

Our choice $P=2 \pi$ gives (41) with the choice

$$
\kappa=\max _{\ell \in[P, P+\theta]} e^{-\ell}(1+2 \ell)=e^{-P}(1+2 P)=\frac{1+4 \pi}{e^{2 \pi}} .
$$

In terms of the notation from Assumptions 3 to 6, one has and one can choose $\bar{k}=0, \mu=2.024, \overline{\mathcal{A}}=|\mathcal{M}|_{\infty}=1.024$, $|\mathcal{B}|_{\infty}=|\mathcal{C}|=1, \overline{\mathcal{L}}=2$, and $\overline{\mathcal{F}}=1$, which give

$$
\bar{k} \tau\left[e^{(\overline{\mathcal{A}}+\bar{k}) \tau}+e^{(\overline{\mathcal{F}}+\bar{k}) \tau}\right] \overline{\mathcal{H}}=0<1
$$

for any choices of $\tau \in(0, \theta)$. One can also compute $\bar{g}=\bar{p}_{1}=\bar{p}_{2}=0$. We deduce that the matrix

$$
\bar{S}=\left[\begin{array}{cc}
0 & 0 \\
\gamma & \frac{1+4 \pi}{e^{2 \pi}}
\end{array}\right]
$$

is Schur stable, so Assumptions 3 to 6 are satisfied for any $\tau \in(0, \pi / 4]$. Thus, we can apply Theorem 2 .

To illustrate our result, Figure 2 shows a MATLAB simulation of the closed-loop system with the controller (49) and the continuous-discrete observer (48) using an integration algorithm of the model (66) with a semi-implicit integration of step 0.001 with initial conditions $x_{1}(0)=x_{2}(0)=-2$ and $\hat{x}_{1}(0)=\hat{x}_{2}(0)=2$, with $\theta=\pi / 4$ and $\tau=0.5$. We again show the estimate $\hat{x}_{2}$ tracking the state component $x_{2}$, and the closed-loop system performance and the control values. Since our simulation shows good stabilization and tracking performance, it helps to illustrate our general theory in the special case of system (66).

\section{6 | CONCLUSIONS}

We provided new constructions of observers and output feedback controls for time-varying nonlinear systems with intermittent output observations and disturbances. Our feedback control result proved exponentially stable convergence of the closed-loop system to the desired equilibrium. This is valuable because it is common in engineering to encounter 
systems for which there are periods during which no output measurements are available for use in the control. The presence of the disturbances and nonlinearities makes the observer design much more challenging than standard observer design problems. Our main strategy to overcome these challenges combined finite time observers and a switched systems approach. Our applications of our theorems to the Mathieu equation exhibited the good performance of our methods in simulations. Many extensions can be expected for systems with delay and for local stability of broader classes of systems.

\section{ORCID}

Frédéric Mazenc (iD) http://orcid.org/0000-0002-6630-3624

Saeed Ahmed (iD) http://orcid.org/0000-0001-9545-9313

Michael Malisoff (iD http://orcid.org/0000-0003-0150-5046

\section{REFERENCES}

1. Cedervall S, Hu X. Nonlinear observers for unicycle robots with range sensors. IEEE Trans Autom Control. 2007;52(7):1325-1329.

2. Dochain D. State and parameter estimation in chemical and biochemical processes: a tutorial. J Process Control. 2003;13(8):801-818.

3. Manchester IR, Andersson KS, Andersson N, Shiriaev AS, Eklund A. A nonlinear observer for on-line estimation of the cerebrospinal fluid outflow resistance. Automatica. 2008;44(5):1426-1430.

4. Perruquetti W, Floquet T, Moulay E. Finite time observers: application to secure communication. IEEE Trans Autom Control. 2008;53(1):356-360.

5. Phanomchoeng G, Rajamani R, Piyabongkarn D. Nonlinear observer for bounded Jacobian systems, with applications to automotive slip angle estimation. IEEE Trans Autom Control. 2011;56(5):1163-1170.

6. Wang W, Nešić D, Postoyan R. Observer design for networked control systems with FlexRay. Automatica. 2017;82:42-48.

7. Bestle D, Zeitz M. Canonical form observer design for non-linear time-variable systems. Int J Control. 1983;38(2):419-431.

8. Hammouri H, Targui B, Armanet F. High gain observer based on a triangular structure. Int J Robust Nonlinear Control. 2002;12(6):497-518.

9. Khalil HK, Praly L. High-gain observers in nonlinear feedback control. Int J Robust Nonlinear Control. 2014;24(6):993-1015.

10. Zemouche A, Boutayeb M. On LMI conditions to design observers for Lipschitz nonlinear system. Automatica. 2013;49(2):585-591.

11. Freidovich LB, Khalil HK. Lyapunov-based switching control of nonlinear systems using high-gain observers. Automatica. 2007;43:150-157.

12. Kazantzis N, Kravaris C. Nonlinear observer design using Lyapunov's auxiliary theorem. Syst Control Lett. 1998;34(5):241-247.

13. Wang L, Astolfi D, Marconi L, Sua H. High-gain observers with limited gain power for systems with observability canonical form. Automatica. 2017;75:16-23.

14. Zeitz M. The extended Luenberger observer for nonlinear systems. Syst Control Lett. 1987;9(2):149-156.

15. Du H, Qian C, Yang S, Li S. Recursive design of finite-time convergent observers for a class of time-varying nonlinear systems. Automatica. 2013;49(2):601-609.

16. Menold PH, Findeisen R, Allgower F. Finite time convergent observers for linear time-varying systems. In: Proceedings of the Mediterranean Conference on Control and Automation; 2003; Rhodes, Greece.

17. Menold PH, Findeisen R, Allgower F. Finite time convergent observers for nonlinear systems. In: Proceedings of the 42nd IEEE Conference on Decision and Control; 2003; Maui, HI.

18. Song Y, Wang Y, Hollway J, Krstic M. Time-varying feedback for finite-time robust regulation of normal-form nonlinear systems. In: Proceedings of the 55th IEEE Conference on Decision and Control; 2016; Las Vegas, NV.

19. Levant A. Robust exact differentiation via sliding mode technique. Automatica. 1998;34(3):379-384.

20. Menard T, Moulay E, Perruquetti W. A global high-gain finite-time observer. IEEE Trans Autom Control. 2010;55(6):1500-1506.

21. Shen Y, Huang Y, Gu J. Global finite time observers for Lipschitz nonlinear systems. IEEE Trans Autom Control. 2011;56(2):418-424.

22. Engel R, Kreisselmeier G. A continuous-time observer which converges in finite time. IEEE Trans Autom Control. 2002;47(7):1202-1204.

23. Raff T, Allgower F. An impulsive observer that estimates the exact state of a linear continuous-time system in predetermined finite time. In: Proceedings of the Mediterranean Conference on Control and Automation; 2007; Athens, Greece.

24. Sauvage F, Guay M, Dochain D. Design of a nonlinear finite-time converging observer for a class of nonlinear systems. J Control Sci Eng. 2007;2007:1-9. Article ID 36954. https://doi.org/10.1155/2007/36954

25. Mazenc F, Fridman E, Djema W. Estimation of solutions of observable nonlinear systems with disturbances. Syst Control Lett. 2015;79:47-58.

26. Shen Y, Huang Y. Uniformly observable and globally Lipschitzian nonlinear systems admit global finite-time observers. IEEE Trans Autom Control. 2009;54(11):2621-2625.

27. Shen Y, Xia X. Semi-global finite-time observers for nonlinear systems. Automatica. 2008;44(12):3152-3156.

28. Yan P, Özbay H. Stability analysis of switched time-delay systems. SIAM J Control Optim. 2008;47(2):936-949. 
29. Yan P, Özbay H, Sansal M. Robust stabilization of parameter varying time delay systems by switched controllers. Appl Comput Math. 2014;13(1):31-45.

30. Mazenc F, Andrieu V, Malisoff M. Design of continuous-discrete observers for time-varying nonlinear systems. Automatica. 2015;57:135-144.

31. Sontag E. Mathematical Control Theory: Deterministic Finite Dimensional Systems. Vol. 6. New York, NY: Springer-Verlag New York; 1998. Texts in Applied Mathematics.

32. Malisoff M, Mazenc F. Constructions of Strict Lyapunov Functions. London, UK: Springer-Verlag London; 2009. Communications and Control Engineering.

33. Mazenc F, Malisoff M. Asymptotic stabilization for feedforward systems with delayed feedback. Automatica. 2013;49(3):780-787.

34. Pearson AE. Nonlinear system identification with limited time data. Automatica. 1979;15(1):73-84.

35. Insperger T, Stépán G. Stability chart for the delayed Mathieu equation. Proc R Soc A Math Phys Eng Sci. 2002;458(2024):1989-1998.

36. Mazenc F, Malisoff M. Trajectory based approach for the stability analysis of nonlinear systems with time delays. IEEE Trans Autom Control. 2015;60(6):1716-1721.

How to cite this article: Mazenc F, Ahmed S, Malisoff M. Finite time estimation through a continuous-discrete observer. Int J Robust Nonlinear Control. 2018;28:4831-4849. https://doi.org/10.1002/rnc.4286

\section{APPENDIX A}

\section{RESULTS FOR MATRICES}

This appendix proves two lemmas, the first of which will be used to prove the second one. The second lemma in this appendix will be used in our proof of Lemma 2 . The following lemma includes the inequality $\left|e^{N}-I\right| \leq e^{|N|}-1$ for any square matrix as the special case where $M=0$.

Lemma 4. The inequality

$$
\left|e^{M+N}-e^{M}\right| \leq\left(1-e^{-|N|}\right) e^{|M|+|N|}
$$

is satisfied for any matrices $M \in \mathbb{R}^{n \times n}$ and $N \in \mathbb{R}^{n \times n}$.

Proof. Let $U_{1}=M$ and $U_{2}=N$. Then, for each pair of integers $(i, j)$ with $i \geq 0$ and $j \geq 1$, there is a function $\lambda_{j, i}:\{1, \ldots, j\} \rightarrow\{1,2\}$ such that $\lambda_{j, 1}(k)=1$ for all $k \in\{1, \ldots, j\}$ and such that

$$
(M+N)^{j}=\sum_{i=1}^{2^{j}} U_{\lambda_{j, i}(1)} \ldots U_{\lambda_{j, i}(j)},
$$

which gives

$$
(M+N)^{j}-M^{j}=\sum_{i=2}^{2^{j}} U_{\lambda_{j, i}(1)} \ldots U_{\lambda_{j, i}(j)} .
$$

It follows that

$$
\left|(M+N)^{j}-M^{j}\right| \leq \sum_{i=2}^{2^{j}}\left|U_{\lambda_{j, i}(1)}\right| \cdots\left|U_{\lambda_{j, i}(j)}\right|=(|M|+|N|)^{j}-|M|^{j} .
$$

Observing that

$$
\left|e^{M+N}-e^{M}\right|=\left|\sum_{j=1}^{+\infty} \frac{(M+N)^{j}-M^{j}}{j !}\right| \leq \sum_{j=1}^{+\infty} \frac{\left|(M+N)^{j}-M^{j}\right|}{j !},
$$

we deduce that

$$
\left|e^{M+N}-e^{M}\right| \leq \sum_{j=1}^{+\infty} \frac{(|M|+|N|)^{j}-|M|^{j}}{j !}=e^{|M|+|N|}-e^{|M|} .
$$

This allows us to conclude. 
Lemma 5. Let $m \geq 1$ be an integer. Let $G \in \mathbb{R}^{n \times n}$ and $v_{i} \in \mathbb{R}^{n \times n}$ for all $i \in\{1, \ldots, m\}$ be matrices such that $|G| \leq \bar{g}$ and $\max _{i}\left|v_{i}\right| \leq \bar{v}$, where $\bar{g}>0$ and $\bar{v}>0$ are two constants. Let $\alpha_{i} \in[0,1]$ be a constant for each $i \in\{1, \ldots, m\}$. Then,

$$
\left|e^{\alpha_{m}\left(G+v_{m}\right)} \cdot e^{\alpha_{m-1}\left(G+v_{m-1}\right)} \ldots . e^{\alpha_{1}\left(G+v_{1}\right)}-e^{\alpha_{\Delta} G}\right| \leq e^{m(\bar{g}+\bar{\nu})}\left(1-e^{-m \bar{\nu}}\right)
$$

is satisfied with the choice $\alpha_{\triangle}=\alpha_{1}+\cdots+\alpha_{m}$.

Proof. We start the proof with some definitions. Set $S(i)=\left(\alpha_{1}+\cdots+\alpha_{i}\right) G$ for all $i \geq 1$, and set

$$
\Omega_{0}=I \text { and } \Omega_{i}=e^{\alpha_{i}\left(G+v_{i}\right)} \cdot e^{\alpha_{i-1}\left(G+v_{i-1}\right)} \ldots e^{\alpha_{1}\left(G+v_{1}\right)} \text { for } i \geq 1 \text {, }
$$

and set $\xi_{0}=0$ and $\xi_{i}=\Omega_{i}-e^{S(i)}$ for all $i \in\{1, \ldots, m\}$. Then, the left side of(A7) is $\left|\xi_{m}\right|$. Consider any $i \in\{1, \ldots, m\}$. Elementary calculations give

$$
\xi_{i}=e^{\alpha_{i}\left(G+v_{i}\right)} \Omega_{i-1}-e^{S(i)}=\left[e^{\alpha_{i}\left(G+v_{i}\right)}-e^{\alpha_{i} G}\right] \Omega_{i-1}+e^{\alpha_{i} G} \xi_{i-1},
$$

and therefore,

$$
\left|\xi_{i}\right| \leq\left|e^{\alpha_{i}\left(G+v_{i}\right)}-e^{\alpha_{i} G}\right|\left|\Omega_{i-1}\right|+e^{\alpha_{i} \bar{g}}\left|\xi_{i-1}\right| \leq\left(1-e^{-\bar{v}}\right) e^{\bar{g}+\bar{v}}\left|\Omega_{i-1}\right|+e^{\bar{g}}\left|\xi_{i-1}\right|,
$$

where the last inequality is a consequence of Lemma 4 and the fact that $\alpha_{i} \in[0,1]$ for all $i$. Since for all $i \in\{0, \ldots, m\}$, we have $\left|\Omega_{i}\right| \leq e^{i(\bar{g}+\bar{v})}$, we obtain

$$
\begin{aligned}
\left|\xi_{m}\right| & \leq\left(1-e^{-\bar{v}}\right) e^{m \beta}+e^{\bar{g}}\left|\xi_{m-1}\right| \\
\left|\xi_{m-1}\right| & \leq\left(1-e^{-\bar{\nu}}\right) e^{(m-1) \beta}+e^{\bar{g}}\left|\xi_{m-2}\right| \\
& \vdots \\
\left|\xi_{1}\right| & \leq\left(1-e^{-\bar{\nu}}\right) e^{\beta}
\end{aligned}
$$

with $\beta=\bar{g}+\bar{v}$. A simple induction argument then gives

$$
\left|\xi_{m}\right| \leq\left(1-e^{-\bar{\nu}}\right) \sum_{k=0}^{m-1} e^{k \bar{g}} e^{(m-k) \beta}=\left(1-e^{-\bar{\nu}}\right) e^{m \beta} \sum_{k=0}^{m-1} e^{-k \bar{\nu}} .
$$

In fact, if we have

$$
\left|\xi_{j}\right| \leq\left(1-e^{-\bar{v}}\right) \sum_{k=0}^{j-1} e^{k \bar{g}} e^{(j-k) \beta}
$$

for some $j \in\{1,2, \ldots, m-1\}$, then (A11) gives

$$
\begin{aligned}
\left|\xi_{j+1}\right| & \leq e^{\bar{g}}\left(1-e^{-\bar{v}}\right) \sum_{k=0}^{j-1} e^{k \bar{g}} e^{(j-k) \beta}+\left(1-e^{-\bar{v}}\right) e^{(j+1) \beta} \\
& =\left(1-e^{-\bar{v}}\right) \sum_{k=1}^{j} e^{k \bar{g}} e^{(j-k+1) \beta}+\left(1-e^{-\bar{v}}\right) e^{(j+1) \beta}=\left(1-e^{-\bar{v}}\right) \sum_{k=0}^{j} e^{k \bar{g}} e^{(j+1-k) \beta},
\end{aligned}
$$

which proves the inductive step. Then, the geometric sum formula implies that inequality (A7) holds.

\section{APPENDIX B}

\section{PROOF OF LEMMA 3}

Set $z=\left(z_{1}, z_{2}\right)^{\top}$. Let $V$ be a positive eigenvector associated with an eigenvalue $\lambda \in[0,1)$ of $\mathcal{M}^{\top}$ (which exist by the Perron-Frobenius theorem, since $\mathcal{M}^{\top}$ is a positive Schur stable matrix). Choose an integer $k \geq 1$ such that $\lambda^{k}<1 / 2$, and set $R=\mathcal{M}^{k}, p=\lambda^{k}$, and $\Psi_{k}(t)=\left(\sup _{s \in[t-k \mathcal{N}, t]} z_{1}(s), \sup _{s \in[t-k \mathcal{N}, t]} z_{2}(s)\right)^{\top}$ for all $t \geq k \mathcal{N}$. Then, (6) can be rewritten as $z(t) \leq$ $\mathcal{M} \Psi_{1}(t)$ (where inequalities of matrices are taken entrywise), and we can prove (by induction on $k$ ) that $z(t) \leq R \Psi_{k}(t)$ for all $t \geq k \mathcal{N}$. We also have $\Psi_{k}(t) \leq 2 \sup _{s \in[t-k \mathcal{N}, t]}\left(z_{1}(s), z_{2}(s)\right)^{\top}$ for all $t \geq k \mathcal{N}$, which follows because $\left(z_{1}\left(s_{1}\right), z_{2}\left(s_{2}\right)\right) \leq$ $\left(z_{1}\left(s_{1}\right), z_{2}\left(s_{1}\right)\right)+\left(z_{1}\left(s_{2}\right), z_{2}\left(s_{2}\right)\right)$ for all $s_{1}$ and $s_{2}$ in $[t-k \mathcal{N}, t]$ and all $t \geq k \mathcal{N}$. Since $V^{\top} R=p V^{\top}$, we conclude that

$$
V^{\top} z(t) \leq p V^{\top} \Psi_{k}(t) \leq 2 p \sup _{s \in[t-k \mathcal{N}, t]} V^{\top} z(s)
$$


for all $t \geq k \mathcal{N}$. Since $2 p \in[0,1)$, we can apply, lemma 1 in the work of Mazenc and Malisoff ${ }^{36}$ (with the choice $w(\ell)=$ $V^{\top} z(\ell+k \mathcal{N})$ ) to find a constant $c_{2}>0$ (that only depends on $k, \mathcal{N}$, and $p$ ) such that $V^{\top} z(t) \leq \sup \left\{V^{\top} z(\ell): 0 \leq \ell \leq\right.$ $k \mathcal{N}\} e^{-c_{2}(t-k \mathcal{N})}$ for all $t \geq k \mathcal{N}$. The lemma now follows because all components of $V$ are positive.

\section{APPENDIX C}

\section{LEMMA ON COMPARISON OF FLOW MAPS}

This appendix provides a lemma that we later use to prove Lemma 2. Consider the system

$$
\dot{X}(t)=N(t) X(t)
$$

with $X$ valued in $\mathbb{R}^{n}$ and where $N:[0,+\infty) \rightarrow \mathbb{R}^{n \times n}$ is a continuous function. Let $\epsilon: \mathbb{R} \rightarrow \mathbb{R}^{n \times n}$ be a piecewise continuous function that is bounded everywhere by some constant $\bar{\epsilon} \geq 0$. Consider

$$
\dot{Y}_{\epsilon}(t)=[N(t)+\epsilon(t)] Y_{\epsilon}(t) .
$$

Let $\Phi$ and $\Phi_{\epsilon}$ denote the fundamental solutions of the systems (C1) and (C2), respectively.

Lemma 6. Let $T_{0}$ and $T \geq T_{0}$ be two real numbers. Let $\delta>0$ be a real number. There exists a constant $\overline{\bar{\epsilon}}>0$ such that if $\bar{\epsilon} \leq \overline{\bar{\epsilon}}$, then, for all $t \in\left[T_{0}, T\right]$ and $s \in\left[T_{0}, t\right]$, the inequality

$$
\left|\Phi(t, s)-\Phi_{\epsilon}(t, s)\right| \leq \delta
$$

holds.

Proof. Observe for later use that the continuity of $\Phi$ and $\Phi^{-1}$ implies that there is a constant $\bar{\Phi} \geq 0$ such that for all $a \in\left[T_{0}, T\right]$ and $b \in\left[T_{0}, a\right]$, we have

$$
\max \left\{\left|\Phi^{-1}(a, b)\right|,|\Phi(a, b)|\right\} \leq \bar{\Phi} .
$$

Set $\lambda_{\epsilon}(a, b)=\Phi^{-1}(a, b) \Phi_{\epsilon}(a, b)-I$. Through simple calculations, we obtain

$$
\begin{aligned}
\frac{\partial \lambda_{\epsilon}}{\partial a}(a, b) & =\frac{\partial \Phi^{-1}}{\partial a}(a, b) \Phi_{\epsilon}(a, b)+\Phi^{-1}(a, b)[N(a)+\epsilon(a)] \Phi_{\epsilon}(a, b) \\
& =-\Phi^{-1}(a, b) N(a) \Phi_{\epsilon}(a, b)+\Phi^{-1}(a, b)[N(a)+\epsilon(a)] \Phi_{\epsilon}(a, b) \\
& =\Phi^{-1}(a, b) \epsilon(a) \Phi_{\epsilon}(a, b)=\Phi^{-1}(a, b) \epsilon(a) \Phi(a, b)\left[\lambda_{\epsilon}(a, b)+I\right],
\end{aligned}
$$

where we also used lemma C.4.1 in the work of Sontag. ${ }^{31}$ Let $v(a, b)=\frac{1}{2}\left|\lambda_{\epsilon}(a, b) V\right|^{2}$, where $V \in \mathbb{R}^{n}$ is any vector satisfying $|V|=1$. Then, the chain rule and (C5) give

$$
\frac{\partial v}{\partial a}(a, b)=V^{\top} \lambda_{\epsilon}(a, b)^{\top} \Phi^{-1}(a, b) \epsilon(a) \Phi(a, b)\left[\lambda_{\epsilon}(a, b)+I\right] V .
$$

Using (C4) and the upper bound of $\epsilon$, we deduce that, for all $t \in\left[T_{0}, T\right]$ and $s \in\left[T_{0}, t\right]$, we have

$$
\frac{\partial v}{\partial t}(t, s) \leq \bar{\Phi}^{2} \bar{\epsilon}\left|\lambda_{\epsilon}(t, s) V\right|^{2}+\bar{\Phi}^{2} \bar{\epsilon}\left|\lambda_{\epsilon}(t, s) V\right|=2 \bar{\Phi}^{2} \bar{\epsilon} v(t, s)+\sqrt{2} \bar{\Phi}^{2} \bar{\epsilon} \sqrt{v(t, s)} .
$$

Using Young's inequality $a b \leq a^{2}+\frac{b^{2}}{4}$ with $b=\sqrt{2}$ and $a=\sqrt{v(t, s)}$, we obtain

$$
\frac{\partial v}{\partial t}(t, s) \leq 3 \bar{\Phi}^{2} \bar{\epsilon} v(t, s)+\frac{1}{2} \bar{\Phi}^{2} \bar{\epsilon} .
$$

By integrating the last inequality between $s$ and $t$ and bearing in mind that $v(s, s)=0$, we deduce that

$$
v(t, s) \leq \frac{1}{6}\left[e^{3 \bar{\Phi}^{2} \bar{\epsilon}(t-s)}-1\right] .
$$

Since (C9) holds for any vector $V$ such that $|V|=1$, we deduce that

$$
\left|\lambda_{\epsilon}(t, s)\right|^{2} \leq \frac{1}{3}\left[e^{3 \bar{\Phi}^{2} \bar{\epsilon}(t-s)}-1\right] .
$$


In addition, $\left|\Phi(t, s)-\Phi_{\epsilon}(t, s)\right| \leq|\Phi(t, s)|\left|I-\Phi(t, s)^{-1} \Phi_{\epsilon}(t, s)\right|$. From the definition of $\lambda_{\epsilon}$ and (C4), we have

$$
\left|\Phi(t, s)-\Phi_{\epsilon}(t, s)\right| \leq|\Phi(t, s)|\left|\lambda_{\epsilon}(t, s)\right| \leq \frac{\bar{\Phi}}{\sqrt{3}} \sqrt{e^{3 \bar{\Phi}^{2} \bar{\epsilon} T}-1} .
$$

We conclude that, if

$$
\bar{\epsilon} \leq \overline{\bar{\epsilon}}=\frac{1}{3 \bar{\Phi}^{2} T} \ln \left(1+\frac{3 \delta^{2}}{\bar{\Phi}^{2}}\right),
$$

then $(\mathrm{C} 3)$ is satisfied.

\section{APPENDIX D}

\section{PROOFS OF LEMMAS 1 AND 2}

\section{D.1 | Proof of Lemma 1}

To prove that the matrix $M+N$ is invertible, let us proceed by contradiction. We suppose that it is not invertible. Then, there is a nonzero vector $V \in \mathbb{R}^{n}$ such that $V^{\top}(M+N)=0$, so invertibility of $M$ gives $V^{\top}=-V^{\top} N M^{-1}$, and so $|V| \leq|V| \bar{m} \bar{n}$. Since $V \neq 0$, we conclude that $1 \leq \bar{m} \bar{n}$, which contradicts (2). We deduce that $M+N$ is invertible. To prove inequality (3), we first set $R=(M+N)^{-1}-M^{-1}$. By multiplying $R$ by $M+N$ and $M$, we obtain $(M+N) R M=M-(M+N)=-N$, and so $M R M=-N-N R M$. We deduce that $R=-M^{-1} N M^{-1}-M^{-1} N R$. As an immediate consequence, we obtain $|R| \leq \bar{m}^{2} \bar{n}+\bar{m} \bar{n}|R|$, which allows us to conclude.

\section{D.2 | Proof of Lemma 2}

Consider any constants $T_{0} \geq 0$ and $T>T_{0}$. We first prove that, for all $a \in\left[T_{0}, T\right]$ and $b \in\left[T_{0}, a\right]$, the inequality

$$
\left|\phi(a, b)-e^{\mathcal{A}(a-b)}\right| \leq|\mathcal{E}|_{\infty}(a-b) e^{\left(|\mathcal{A}|+|\mathcal{E}|_{\infty}\right)(a-b)}
$$

holds. Recalling that $\mathcal{E}$ is locally Lipschitz, let $K_{\mathcal{E}} \geq 0$ be a Lipschitz constant for $\mathcal{E}(t)$ on $\left[T_{0}, T\right]$. Let $r>T-T_{0}$ be a positive integer to be selected later. We define an increasing sequence of real numbers $t_{i}$ by $t_{0}=T_{0}$ and $t_{i+1}-t_{i}=p_{*}$, where $p_{*}=\left(T-T_{0}\right) / r$. Let $\sigma_{r}$ be a switching sequence defined by $\sigma_{r}(t)=t_{i}$ when $t \in\left[t_{i}, t_{i+1}\right)$ for all integers $i \geq 0$. Then,

$$
\left|\mathcal{E}(u)-\mathcal{E}\left(\sigma_{r}(u)\right)\right| \leq K_{\mathcal{E}}\left|u-\sigma_{r}(u)\right| \leq K_{\mathcal{E}} p_{*}=K_{\mathcal{E}} \frac{T-T_{0}}{r}
$$

for all $u \in\left[T_{0}, T\right]$.

Consider any constant $\delta>0$. Let $\phi_{r}$ denote the fundamental solution of the time-varying linear system $\dot{\xi}(t)=[\mathcal{A}+$ $\left.\mathcal{E}\left(\sigma_{r}(t)\right)\right] \xi(t)$. By letting $r \rightarrow+\infty$ in (D2) and using Lemma 6 (with the choices $N(t)=A+\mathcal{E}(t)$ and $\epsilon(t)=\mathcal{E}\left(\sigma_{r}(t)\right)-\mathcal{E}(t)$ ), we deduce that there is an integer $r_{c}>0$ such that, when $r \in \mathbb{N}$ is such that $r \geq r_{c}$, then

$$
\left|\phi\left(a_{1}, a_{2}\right)-\phi_{r}\left(a_{1}, a_{2}\right)\right| \leq \delta
$$

for all $a_{1} \in\left[T_{0}, T\right]$ and $a_{2} \in\left[T_{0}, a_{1}\right]$. Fix two constants $t \in\left(T_{0}, T\right]$ and $s \in\left[T_{0}, t\right)$. From D3, it follows that

$$
\left|\phi(t, s)-e^{\mathcal{A}(t-s)}\right| \leq\left|\phi(t, s)-\phi_{r}(t, s)\right|+\left|\phi_{r}(t, s)-e^{\mathcal{A}(t-s)}\right| \leq \delta+\left|\Lambda_{r}(t, s)\right|
$$

with $\Lambda_{r}(t, s)=\phi_{r}(t, s)-e^{\mathcal{A}(t-s)}$.

Since $t \in\left(T_{0}, T\right]$ and $s \in\left[T_{0}, t\right)$, there exists $l \in \geq 0$ such that $t \in\left[t_{l}, t_{l+1}\right)$ and there exists $p \in \geq 0$ such that $s \in\left[t_{p}, t_{p+1}\right)$ with $p \leq l$. If $p=l$, then

$$
\left|\Lambda_{r}(t, s)\right|=\left|\phi_{r}(t, s)-e^{\mathcal{A}(t-s)}\right|=\left|e^{(t-s)\left(\mathcal{A}+\mathcal{E}\left(t_{l}\right)\right)}-e^{\mathcal{A}(t-s)}\right| .
$$

From Lemma 4, it follows that

$$
\left|\Lambda_{r}(t, s)\right| \leq\left(1-e^{-(t-s)|\mathcal{E}|_{\infty}}\right) e^{(t-s)\left(|\mathcal{A}|+|\mathcal{E}|_{\infty}\right)} .
$$


On the other hand, if $p<l$, then we obtain

$$
\begin{aligned}
\Lambda_{r}(t, s) & =\phi_{r}\left(t, t_{l}\right) \phi_{r}\left(t_{l}, t_{l-1}\right) \ldots \phi_{r}\left(t_{p+1}, s\right)-e^{\mathcal{A}(t-s)} \\
& =e^{\left(t-t_{l}\right)\left(\mathcal{A}+\mathcal{E}\left(t_{l}\right)\right)} e^{p_{*}\left(\mathcal{A}+\mathcal{E}\left(t_{l-1}\right)\right)} \ldots e^{p_{*}\left(\mathcal{A}+\mathcal{E}\left(t_{p+1}\right)\right)} e^{\left(t_{p+1}-s\right)\left(\mathcal{A}+\mathcal{E}\left(t_{p}\right)\right)}-e^{\mathcal{A}(t-s)} \\
& =e^{\frac{t-t_{l}}{p_{*}} p_{*}\left(\mathcal{A}+\mathcal{E}\left(t_{l}\right)\right)} e^{p_{*}\left(\mathcal{A}+\mathcal{E}\left(t_{l-1}\right)\right)} \ldots e^{p_{*}\left(\mathcal{A}+\mathcal{E}\left(t_{p+1}\right)\right)} e^{\frac{t_{p+1}-s}{p_{*}} p_{*}\left(\mathcal{A}+\mathcal{E}\left(t_{p}\right)\right)}-e^{\mathcal{A}(t-s)} .
\end{aligned}
$$

Since $\left(t-t_{l}\right) / p_{*},\left(t_{p+1}-s\right) / p_{*}$, and $p_{*}$ are all contained in $[0,1]$, it follows from Lemma 5 with the choices $m=\ell-p+1$, $G=p_{*} \mathcal{A}$, and $v_{i}=p_{*} \mathcal{E}\left(t_{i}\right)$ that

$$
\begin{aligned}
\left|\Lambda_{r}(t, s)\right| & \leq e^{(\ell-p+1) p_{*}\left(|\mathcal{A}|+|\mathcal{E}|_{\infty}\right)}\left(1-e^{-(\ell-p+1) p_{*}|\mathcal{E}|_{\infty}}\right) \\
& \leq e^{2 p_{*}\left(|\mathcal{A}|+|\mathcal{E}|_{\infty}\right)} e^{(t-s)\left(|\mathcal{A}|+|\mathcal{E}|_{\infty}\right)}\left(1-e^{-2 p_{*}\left(|\mathcal{A}|+|\mathcal{E}|_{\infty}\right)} e^{-(t-s)|\mathcal{E}|_{\infty}}\right),
\end{aligned}
$$

where we used the fact that $(\ell-p-1) p_{*} \leq t-s$. This inequality and (D4) give

$$
\left|\phi(t, s)-e^{\mathcal{A}(t-s)}\right| \leq \delta+e^{2 p_{*}\left(|\mathcal{A}|+|\mathcal{E}|_{\infty}\right)} e^{(t-s)\left(|\mathcal{A}|+|\mathcal{E}|_{\infty}\right)}\left(1-e^{-2 p_{*}\left(|\mathcal{A}|+|\mathcal{E}|_{\infty}\right)} e^{-(t-s)|\mathcal{E}|_{\infty}}\right) .
$$

Since $\delta>0$ is arbitrary and since we can make $r$ as large as we wish (and therefore, $p_{*}$ as small a positive value as we wish), we deduce from (D9) that

$$
\left|\phi(t, s)-e^{\mathcal{A}(t-s)}\right| \leq e^{(t-s)\left(|\mathcal{A}|+|\mathcal{E}|_{\infty}\right)}\left(1-e^{-(t-s)|\mathcal{E}|_{\infty}}\right)
$$

for all $t \in\left(T_{0}, T\right]$ and $s \in\left[T_{0}, t\right)$. Since the constants $T$ and $T_{0}$ are arbitrary, we can conclude because $1-e^{-s} \leq s$ for all $s \geq 0$. 